\title{
45Sc Solid State NMR Spectroscopy - A Complementary Tool to X-ray Crystallography for Structure Determination of Intermetallic Com-pounds
}

\begin{tabular}{|r|l|}
\hline Journal: & Zeitschrift für Anorganische und Allgemeine Chemie \\
\hline Manuscript ID: & zaac. 201000197.R1 \\
\hline Wiley - Manuscript type: & Research Report \\
\hline Date Submitted by the \\
Author: & $19-$ May-2010 \\
\hline Complete List of Authors: & $\begin{array}{l}\text { Poettgen, Rainer; Westf. Wilhelms-Universitaet, Inst. f. Anorgan. u. } \\
\text { Analyt. Chemie } \\
\text { Eckert, Hellmut }\end{array}$ \\
\hline Keywords: & scandium, solid state NMR \\
\hline
\end{tabular}

\section{S) ScholaroNE \\ Manuscript Central}




\title{
${ }^{45}$ Sc Solid State NMR Spectroscopy - A Complementary Tool to X-ray Crystallography for Structure Determination of Intermetallic Com- pounds
}

\author{
Hellmut Eckert* ${ }^{[a]}$ and Rainer Pöttgen* ${ }^{[b]}$
}

Received,

Running title: ${ }^{45} \mathrm{Sc}$ Solid State NMR

Keywords: $\quad{ }^{45}$ Sc; Solid NMR Spectroscopy; Intermetallic Compounds

\begin{abstract}
Sc solid state NMR is a useful complementary tool to x-ray crystallographyfor structure determination. The recent developments in the field of intermetallic scandium compounds are reviewed. The structural topics concern defect formation, structural disorder, superstructure formation, as well as the detection of multiple scandium sites. To address questions of this kind, high-resolution ${ }^{45} \mathrm{Sc}$ NMR techniques have been established as a new structural tool for the quantification of the local coordination scandium environments and the detailed characterization of their internal NMR interaction parameters. Owing to the moderately sized nuclear electric quadrupole moment of ${ }^{45} \mathrm{Sc}$, the magic-angle spinning spectra are affected both by first- and second-order quadrupolar perturbations, which can be analyzed and quantified by fast magic angle spinning, assisted by lineshape simulations. The resolution can be further improved by correlating triple quantum (TQ) with single quantum coherences. Using this 2D NMR methodology, the effects of magnetic shielding and second-order quadrupolar perturbations on the MAS NMR lineshape $\mathrm{cn}$ be separated, enabling a more precise experimental characterization of electric field gradients present at the ${ }^{45} \mathrm{Sc}$ nuclei. Structure validation includes the precise measurement of these parameters and comparison with quantum mechani-
\end{abstract}


cally calculated values based on the atomic positions. In this regard, satisfactory results have been obtained using the WIEN2k programme.

* Prof. Dr. Rainer Pöttgen

Fax: +49-251-8336002

E-mail: pottgen@uni-muenster.de

Prof. Dr. Hellmut Eckert

Fax: +49-251-8329159

E-mail: eckerth@uni-muenster.de

[a] Institut für Anorganische und Analytische Chemie, Universität Münster

Corrensstrasse 30, 48149 Münster, Germany

[b] Institut für Physikalische Chemie, Universität Münster

Corrensstrasse 30, 48149 Münster, Germany

\section{Introduction}

Among the rare earth metals the element scandium sets itself apart by being the one having the smallest atomic size. This has clear consequences for the crystal chemistry of intermetallic compounds. While in many cases the latter are isotypic with the corresponding lutetium compounds, in many other cases the $\mathrm{Sc}^{3+}$ ions are too small to realize the same atomic arrangements. Small structural distortions are often the consequence, resulting in the formation of compounds with deficient site occupancies, with superstructures derived from those of larger rare-earth analogues or crystallizing in other, unprecedented structure types. The crystal chemistry of scandium-based intermetallic compounds has been summarized in two reviews [1,2]. To characterize the abovementioned structural peculiarities the availability of structural information at the local level is of great complementary value to single-crystal x-ray diffraction data. During the past decade, we have been developing the potential of high-resolution multinuclear solid state nuclear magnetic resonance (NMR) spectroscopy as an element-selective, sitespecific and inherently quantitative tool for the detailed structural characterization of intermetallic compounds.

Scandium intermetallic compounds are attractive candidates for such studies, as the ${ }^{45} \mathrm{Sc}$ isotope $(\mathrm{I}=7 / 2)$ has $100 \%$ natural abundance, a moderately high gyromagnetic ratio of $6.50880 \times 10^{7} \mathrm{rad} \mathrm{T}^{-1} \mathrm{~s}^{-1}$, and a medium-sized nuclear electric quadrupole moment $Q=0.22 \times 10^{-28} \mathrm{~m}^{2}$. Although these characteristics are excellent pre-requisites for 
solid state NMR experiments, only few NMR spectroscopic reports can be found in the literature and little is known about the correlation of the ${ }^{45} \mathrm{Sc}$ NMR parameters with local structural data.

One main problem in the early stages of rare earth chemistry was the commercial availability of scandium. Only few scandium minerals like thortveitit $\mathrm{Sc}_{2} \mathrm{Si}_{2} \mathrm{O}_{7}$ or sterretite $\mathrm{ScPO}_{4} \cdot 2 \mathrm{H}_{2} \mathrm{O}$ are known. Furthermore scandium is found in highly dispersed form $(<1 \%)$ in wolframite and cassiterite and, due to a similar radius, also in several uranium minerals. Modern liquid-liquid extraction techniques of suitable complexes have made scandium now more disposable at a reasonable price.

In the past most ${ }^{45} \mathrm{Sc}$ solid state NMR studies have been performed on diverse complex oxides, zeolite-based materials, glasses [3-23], organic-inorganic hybrid materials [24], doped lanthanum fluoride nanoparticles [25], antiferromagnetic thiospinels [26, 27], and fullerenes [28-32]. Besides standard methodology, a variety of modern techniques like multi-quantum magic angle spinning NMR have been applied to diverse oxidic scandium compounds [33-36] in order to get more detailed structural information on these materials, e. g. differentiation of the scandium sites in organically templated scandium fluorophosphate frameworks [37], to work out the influence of the coordination number on the isotropic chemical shift in stabilized zirconia [38] and to systemize the ${ }^{45} \mathrm{Sc}$ chemical shifts in several basic inorganic scandium compounds [39].

When it comes to ${ }^{45} \mathrm{Sc}$ solid state NMR on intermetallic compounds only few data are available in the literature. Several studies have been performed on the complex silicides $\mathrm{Sc}_{5} T_{4} \mathrm{Si}_{10}(T=\mathrm{Co}, \mathrm{Rh}, \mathrm{Ir})$ [40-42]. The NMR data of $\mathrm{Sc}_{5} \mathrm{Ir}_{4} \mathrm{Si}_{10}$ were used as a baseline measurement to illustrate the anomalous behaviour of the isotypic lutetium compounds [40]. More detailed measurements on $\mathrm{Sc}_{5} \mathrm{Co}_{4} \mathrm{Si}_{10}$ [41] showed a near zero value of the ${ }^{45} \mathrm{Sc}$ Knight shift, suggesting almost empty $d$ orbitals of the scandium atoms. Such a behaviour is in agreement with a description $\left(\mathrm{Sc}_{5}\right)^{\delta+}\left[\mathrm{Co}_{4} \mathrm{Si}_{10}\right]^{\delta-}$, underlining the polyanionic character of the three-dimensional cobalt-silicon network. The three crystallographically independent scandium sites in $\mathrm{Sc}_{5} T_{4} \mathrm{Si}_{10}$ can clearly be resolved in the ${ }^{45}$ Sc spectra [42]. 
Besides enabling the resolution of crystal chemically different sites, ${ }^{45} \mathrm{Sc}$ magnetic shieldings are also able to detect small changes in electron density around the scandium nuclei. This has been demonstrated recently for the boride $\operatorname{ScRh}_{3} B_{x}[43,44]$ with filled $\mathrm{Cu}_{3} \mathrm{Au}$ type structure. The ${ }^{45} \mathrm{Sc}$ resonance frequency decreases with increasing boron concentration. Similarly tiny effects have also been observed for $\alpha-\mathrm{ScH}_{\mathrm{x}}(\mathrm{x}=0,0.11$, $0.27)$ and $\alpha-\mathrm{ScD}_{\mathrm{x}}(\mathrm{x}=0.05,0.22)$ [45]. The unusual isotope effect between the hydride and the deuteride results in different ${ }^{45} \mathrm{Sc}$ relaxation rates; for comparable $\mathrm{x}$ values, the peak amplitude in $\alpha-\mathrm{ScD}_{\mathrm{x}}$ is found to be significantly higher than in $\alpha-\mathrm{ScH}_{\mathrm{x}}$. The hydrogen storage material $\mathrm{Mg}_{0.65} \mathrm{Sc}_{0.35} \mathrm{H}_{2.2}$ [46] shows magnesium-scandium distribution in the $f c c$ structure. Using a double-quantum NMR technique with ${ }^{45} \mathrm{Sc}$ recoupling two types of deuterium atoms with and without scandium neighbours could be differentiated. ${ }^{2} \mathrm{H}-\left\{{ }^{45} \mathrm{Sc}\right\}$ TRAPDOR NMR indicates a non-statistical magnesium and scandium distribution, indicating a complex, not yet known superstructure.

During the past five years, within SPP 1166 Lanthanoidspezifische Funktionalitäten in Molekül und Material we started a more systematic investigation and studied the structural and ${ }^{45} \mathrm{Sc}$ solid state NMR spectroscopic data of various intermetallic scandium compounds $\mathrm{Sc}_{\mathrm{x}} T_{\mathrm{y}} X_{\mathrm{z}}\left(T=\right.$ late transition metal; $X=$ element of the $3^{\text {rd }}, 4^{\text {th }}$, or $5^{\text {th }}$ main group) [47-58] with respect to scandium site assignment, defect formation and structural disorder. These results are reviewed herein.

\section{Fundamental aspects and experimental methodology of ${ }^{45} \mathrm{Sc}$ MAS- NMR}

NMR spectra of the ${ }^{45} \mathrm{Sc}$ isotope are prominently influenced by magnetic shielding and nuclear electric quadrupolar coupling perturbations. Magnetic shielding effects comprise contributions from a local term ("chemical shift") and a term arising from the nuclear spin interactions with unpaired spin density associated with the conduction electrons ("Knight shift"). These contributions cannot be separated and only the combined magnetic shielding interaction is measured. Nuclear electric quadrupolar coupling perturbations originate from the interaction of non-spherical charge distribution of the ${ }^{45} \mathrm{Sc} \mathrm{nu}$ cleus (quantified in terms of its nuclear electric quadrupole moment) with the electric 
field gradient (EFG) created by its local environment. The EFG is a symmetric secondrank tensor, which can be diagonalized in a molecular axis system. The sum of the diagonal elements $\mathrm{q}_{\mathrm{xx}}+\mathrm{q}_{\mathrm{yy}}+\mathrm{q}_{\mathrm{zz}}$ vanishes (Laplace equation) so that the interaction can be described in terms of two parameters, the nuclear electric quadrupolar coupling constant $\mathrm{C}_{\mathrm{Q}}=\mathrm{e}^{2} \mathrm{q}_{\mathrm{zz}} \mathrm{Q} / \mathrm{h}$ (product of the quadrupolar moment and the principal value $\mathrm{q}_{\mathrm{zz}}$ of the electric field gradient tensor) and the asymmetry parameter

$$
\eta_{\mathrm{Q}}=\left(\mathrm{q}_{\mathrm{xx}}-\mathrm{q}_{\mathrm{yy}}\right) / \mathrm{q}_{\mathrm{zz}}
$$

$\left(0 \leq \eta_{\mathrm{Q}} \leq 1\right)$, which characterizes the deviation of the EFG from cylindrical symmetry.

The quadrupolar interaction competes with the Zeeman interaction for the spin alignment and the mathematical treatment is particularly straightforward if one interaction dominates. In the case of ${ }^{45} \mathrm{Sc}$ at customary magnetic flux densities (4.7 to $14.6 \mathrm{~T}$ ) the Zeeman interaction dominates and the effect of the nuclear electric quadrupolar interaction upon the spectra can be calculated within several levels of perturbation theory. For moderately weak quadrupolar coupling $\left(\mathrm{C}_{\mathrm{Q}}<0.05 v_{\mathrm{L}}\right)\left(v_{\mathrm{L}}\right.$ being the ${ }^{45} \mathrm{Sc}$ Zeeman frequency) first-order perturbation theory suffices for computing the energy corrections to the Zeeman levels. The relevant interaction Hamiltonian takes the form

$$
\mathcal{H}_{Q}^{(1)}=\frac{C_{Q}}{2 I(2 I-1)} \cdot \frac{1}{2}\left(3 \cos ^{2} \theta-1-\eta_{Q} \sin ^{2} \theta \cos 2 \phi\right) \cdot \frac{1}{2}\left[3 \hat{I}_{z}^{2}-I(I+1)\right]
$$

where $\theta$ and $\phi$ are the polar angles specifying the orientation of the EFG tensor relative to the magnetic field direction. For each Zeeman state $\mid \mathrm{m}>$ the corresponding energy corrections $\mathrm{E}_{\mathrm{m}}{ }^{(1)}$ are computed from the integrals $\left\langle\mathrm{m}\left|\mathbf{H}_{\mathbf{Q}}\right| \mathrm{m}>\right.$. As the energy corrections are proportional to $\mathrm{m}^{2}$, the energy level shifts of the $\mid+1 / 2>$ and the $\mid-1 / 2>$ states are identical at each orientation, and the width of the central $|+1 / 2><->|-1 / 2>$ transition is unaffected by the quadrupole interaction. In contrast, the frequencies of all the other $\mid \pm m><->$ $\pm(m-1)>$ transitions are orientationally dependent, giving rise to anisotropically broadened satellite transitions in polycrystalline samples (see Figure 1). Under MAS conditions these satellite transitions generate a spinning sideband manifold, the envelope of which approximates the static line shape. By comparing the experimental pattern mani- 
fold with simulated spectra, the parameters $C_{Q}$ and $\eta$ can be determined in principle. The simulations shown in Figure 2 illustrate that the intensity distribution of the sideband patterns is quite sensitive to the value of the asymmetry parameter $\eta$. In practice the sideband intensity profile offers satisfactory results only for relatively small electric field gradients $\left(\mathrm{C}_{\mathrm{Q}}<5 \mathrm{MHz}\right)$. For larger values the amplitudes of the individual spinning sidebands are seriously affected by the inherent limitations of the probe bandwidths and by the excitation windows afforded by radio frequency pulses of finite length. In addition, probe "dead time" problems necessitating delayed signal acquisition produce large first-order phase shifts at large resonance offset effects resulting in baseline artifacts and phase twists that cannot always be corrected perfectly. Finally, inevitably occurring small fluctuations in MAS speeds (on the order of a few $\mathrm{Hz}$ ) lead to significant broadening of higher order spinning sidebands, affecting their amplitudes. All of these problems lead to imprecisions in the experimental data making the comparison with simulated MAS manifolds difficult for larger quadrupolar coupling constants $\left(\mathrm{C}_{\mathrm{Q}}>5\right.$ $\mathrm{MHz}$ ), unless elaborate software corrections for these effects are made. Thus, the analysis of MAS-spinning sideband patterns is generally not used for spectra affected by stronger quadrupolar couplings and one resorts to alternative methods, vide infra.

To calculate the effect of stronger quadrupolar couplings on the NMR spectra beyond the limit of $\mathrm{C}_{\mathrm{Q}}<0.05 v_{\mathrm{L}}$ the perturbation approach has to be extended to second order. In this case, also the central $|1 / 2><->|-1 / 2>$ transitions, are anisotropically broadened. Furthermore, the orientational dependence of the energy levels is more complicated than given by eq. $\{2\}$, as a result of which the resulting line broadening effects in polycrystalline samples cannot be completely eliminated by MAS. Rather, the MASNMR spectra yield characteristic line shapes, from which $\mathrm{C}_{\mathrm{Q}}$ and $\eta$ can be extracted by simulation. In practice this method is limited whenever additional line broadening mechanisms (such as dipolar interactions or distribution effects of magnetic shielding and quadrupolar coupling in case of disorder) contribute to the line width to a significant extent. Our experience has shown that in intermetallic compounds second-order quadrupolar broadening generally dominates if $\mathrm{C}_{\mathrm{Q}}\left({ }^{45} \mathrm{Sc}\right)>9 \mathrm{MHz}$ at a magnetic flux density of 11.7 $\mathrm{T}$, producing well-resolved line shape features that can be compared straightforwardly to line shape simulations. ${ }^{45} \mathrm{Sc}$ quadrupolar interactions of intermediate strength 
( 5 to $9 \mathrm{MHz}$ ) can be best characterized by the triple-quantum (TQ) MAS experiment [59]. The latter technique represents a powerful approach not only for removing second order quadrupolar broadening effects producing highly resolved spectra, but also for characterizing the nuclear electric quadrupolar interaction in more detail. The centers of gravity of the signals observed in the isotropic (F1-) and the anisotropic (F2-) dimensions of this experiment, $\delta_{\mathrm{F} 1}$ and $\delta_{\mathrm{F} 2}$ are given by:

$$
\begin{array}{ll} 
& \delta_{\mathrm{F} 1}=\left(34 \delta_{\mathrm{ms}}{ }^{\text {iso }}-60 \delta_{\mathrm{Q}}\right) / 9 \\
& \delta_{\mathrm{F} 2}=\delta_{\mathrm{ms}}^{\text {iso }}+3 \delta_{\mathrm{Q}} \\
& \delta_{\mathrm{Q}}=(\mathrm{SOQE})^{2} /\left(30 v_{\mathrm{L}}^{2}[2 \mathrm{I}(2 \mathrm{I}-1)]^{2}\right)
\end{array}
$$

and $\delta_{\mathrm{ms}}$ iso is the isotropic magnetic shielding constant (often loosely called "chemical shift" when measured against a reference sample). In the above expression $v_{\mathrm{L}}$ is the nuclear Larmor frequency, and the parameter SOQE, the so-called second order quadrupolar effect is given by the relation:

$$
\mathrm{SOQE}=\mathrm{C}_{\mathrm{Q}}\left(1+\eta_{\mathrm{Q}}^{2} / 3\right)^{1 / 2}
$$

Thus, the analysis of the centers of gravity in both dimensions of a TQMAS experiment affords the isotropic chemical shift $\delta^{\text {cs }}$ iso and the parameter SOQE. Unfortunately, however, the latter cannot separate the principal value and the asymmetry parameter of the nuclear electric quadrupolar coupling tensor.

Theoretical ab-initio values are available from optimized molecular geometries or crystal structure data using programs such as the WIEN2k code [60]. This code is a fullpotential all electron method based on the LAPW + LO method and has proven very suitable for calculating the electric field gradient properties of intermetallic compounds.

\section{Experimental}

The $\mathrm{Sc}_{\mathrm{x}} T_{\mathrm{y}} X_{\mathrm{z}}$ compounds discussed herein can easily be synthesized from the pure elements either by arc- or by induction melting under purified argon. For homogenization of the samples annealing in sealed evacuated silica tubes can be performed for sev- 
eral days. Single crystals of ScNiSn and ScPdSn [53] were obtained from a tin flux. For further experimental details we refer to the original literature. Standard characterization of the samples was performed via powder X-ray diffraction and metallography in combination with EDX.

${ }^{45} \mathrm{Sc}$ MAS NMR spectra were recorded at ambient temperature at a resonance frequency of $121.5 \mathrm{MHz}$ on a Bruker DSX-500 spectrometer, operating a 2.5 mm MAS NMR probe at a MAS rotation frequency of $25 \mathrm{kHz}$. Pulse lengths of typically 0.2 to 1.0 $\mu$ s $\left(20^{\circ}\right.$ solid flip angle) were used with a recycle delay of $0.5 \mathrm{~s}$. The RF field strength corresponds to a nutation frequency of about $85 \mathrm{kHz}$ for the $0.2 \mathrm{M}$ scandium nitrate aqueous solution reference. The lineshape simulation programme DMFIT [61] was used for extracting the isotropic magnetic shielding and nuclear electric quadrupolar coupling parameters from the MAS-NMR lineshapes. 2-D TQMAS correlation spectra were obtained using the three-pulse, zero-quantum filtering variant and processed using the shearing technique. Further details are available in the original literature. Density functional theory (DFT) calculations of the electric field gradient parameters were done using the WIEN2k programme [60]. Details of the calculational parameters are given in the individual publications. Generally, the atomic positions from the structure solutions were used directly, without further optimization.

\section{Structural data}

The crystal chemical data and chemical bonding features of the diverse intermetallic scandium compounds have been discussed in detail in the original work. In this chapter we focus on the local scandium coordination environments which are presented in Figures 3-7. Most compounds investigated in the last years have the simple ScTX composition, however, depending on the valence electron concentration (VEC) and the respective radii of the $T$ and $X$ component, they adopt different structure types.

ScAuGe and ScAuSi [50, and refs. therein] are superstructures of the $\mathrm{AlB}_{2}$ type. The gold and silicon (germanium) atoms build up puckered $\mathrm{Au}_{3} \mathrm{Si}_{3}$ and $\mathrm{Au}_{3} \mathrm{Ge}_{3}$ hexagons which coordinate to the scandium atoms (Figure 3). Due to a different puckering (AB in $\mathrm{ScAuGe}$ and $\mathrm{AA}^{\prime}$ in $\mathrm{ScAuSi}$ ) one obtains one scandium coordination in the germanides but two crystallographically independent ones in the silicide; Sc1 with nearer silicon and 
Sc2 with nearer gold neighbors. These coordinations can be distinguished in the NMR experiment (vide infra). ScCuSn is isotypic with $\mathrm{ScAuGe}$.

A stacking sequence $\mathrm{ABC}$ for the $\mathrm{Au}_{3} \mathrm{Sn}_{3}$ hexagons occurs in the cubic structure of ScAuSn [47, and refs. therein] (Figure 3). These hexagons show stronger puckering and the coordination number for the scandium atoms is reduced to 10 with four gold and six tin neighbors. The antimonides $\mathrm{ScTSb}(T=\mathrm{Ni}, \mathrm{Pd}, \mathrm{Pt})$ adopt the same MgAgAs structure type. In contrast to the stannide we observed small defects on some transition metal sites of the antimonides. These defects depend on the thermal treatment of the samples and they affect the electronic environment of the scandium atoms (see below). Only $\mathrm{ScPtSb}$ showed no defect formation.

A switch in structure type is observed for the stannides $\mathrm{ScPdSn}, \mathrm{ScPtSn}$ [51, and refs. therein] and ScAgSn [49] (Figure 4). They crystallize with pronounced hexagonal, ZrNiAl type subcells, however, weak additional reflections show superstructure formation which accounts for the small differences in the radii of the transition metal atoms and the small differences in VEC. The main difference between the ScPdSn and ScPtSn on the one and ScAgSn on the other hand concerns the transition metal-tin coloring which switches between the group 10 and 11 transition metals. In ScAgSn each scandium atoms is sandwiched by two $\mathrm{Pd}_{2} \mathrm{Sn}_{3}\left(\mathrm{Ag}_{3} \mathrm{Sn}_{2}\right)$ pentagons which are connected by a palladium (tin atom). Six scandium atoms complete the coordination shell. Another major difference concerns the distance between the pentagons. In going from ScPdSn to ScAgSn (i. e. with increasing VEC) we observe a large increase of the subcell $c$ parameter (distance between the hexagons) from 353.7 to $433.9 \mathrm{pm}$. This has a drastic effect on the interatomic Sc-Sc distances. Upon superstructure formation one loses the subcell mirror planes within the pentagons and the latter become slightly puckered as is readily visible from Figure 4. Larger distortions in the scandium substructure are also evident from the Sc2 and Sc3 polyhedra. The key to solve the ScAgSn superstructure was interplay of high-quality single crystal X-ray data in combination with ${ }^{45} \mathrm{Sc}$ solid state NMR (vide infra). Since silver and tin differ by only three electrons, the difference in X-ray scattering power is not large.

Isoelectronic ScNiSn [53] with the lighter nickel atoms crystallizes with the TiNiSi type structure, an orthorhombically distorted $\mathrm{AlB}_{2}$ superstructure. Two ordered and 
tilted $\mathrm{Ni}_{3} \mathrm{Sn}_{3}$ hexagons coordinate the scandium atoms (Figure 5). The single scandium sites could be well resolved in the ${ }^{45} \mathrm{Sc}$ spectrum. All sites in the $\mathrm{ScNiSn}$ structure are fully occupied and the single crystal X-ray data gave no hint for disorder. This is different for the cubic stannide $\mathrm{ScNi}_{2} \mathrm{Sn}$ [51] and for the silicide $\mathrm{ScNiSi}_{3}$ [54]. The scandium atoms in $\mathrm{ScNi}_{2} \mathrm{Sn}$ have a CsCl-related nickel coordination. Single crystal X-ray data for two crystals from different samples revealed significant defects on the nickel sites, leading to the refined compositions $\mathrm{ScNi}_{1.54} \mathrm{Sn}$ and $\mathrm{ScNi}_{1.85} \mathrm{Sn}$. These defects have drastic effect on the local scandium coordination and thus on the ${ }^{45} \mathrm{Sc}$ NMR spectra [51].

Similar behaviour is observed for $\mathrm{ScNiSi}_{3}$ [54]. This structure had previously been reported with a non-centrosymmetric space group. Single crystal X-ray and also the ${ }^{45} \mathrm{Sc}$ solid state NMR data clearly pointed to centrosymmetricity. The scandium coordination (Figure 5) consists of two planar $\mathrm{Ni}_{2} \mathrm{Si}_{4}$ hexagons which are bridged by two further silicon atoms, leading to coordination number 14. The four nickel neighbours form a rectangle around the scandium atom. An important result from the single crystal X-ray work concerns the nickel content of this silicide. Refinements of the occupancy parameters on two crystals pointed to significant defect formation with refined compositions $\mathrm{ScNi}_{0.913} \mathrm{Si}_{3}$ and $\mathrm{ScNi}_{0.929} \mathrm{Si}_{3}$. A local picture for the scandium coordination then leads to a significant portion of scandium atoms which have reduced coordination number 13 , where statistically one nickel position remains unoccupied. The ${ }^{45} \mathrm{Sc}$ spectroscopic consequences are discussed in more detail below.

The scandium coordinations in the complex silicides $\mathrm{Sc}_{2} \mathrm{RuSi}_{2}$ [56] and $\mathrm{Sc}_{3} \mathrm{RuSi}_{3}$ [57] are presented in Figure 6. $\mathrm{Sc}_{2} \mathrm{RuSi}_{2}$ adopts the monoclinic $\mathrm{Zr}_{2} \mathrm{CoSi}_{2}$ structure and one observes two crystallographically independent scandium sites with very low symmetric coordination. Even more complex crystal chemistry occurs for $\mathrm{Sc}_{3} \mathrm{RuSi}_{3}$ which crystallizes with a new structure type, also observed with rhodium, iridium, and platinum as the transition metal component. This silicide contains three different scandium positions, again with a low-symmetrical arrangement. In both structures the very asymmetric coordination concerns the ruthenium-silicon polyanion as well as the scandium neighbours. The bonding pattern in both silicides is quite similar. One observes strong covalent $\mathrm{Ru}-\mathrm{Si}$ and $\mathrm{Si}-\mathrm{Si}$ bonding within the $\left[\mathrm{RuSi}_{2}\right]^{\delta-}$ and $\left[\mathrm{RuSi}_{3}\right]^{\delta-}$ polyanions. 
The new platinum-rich silicide $\mathrm{Sc}_{4} \mathrm{Pt}_{7} \mathrm{Si}_{2}$ [58] is a further example for a compound with two scandium sites. Here we observe distinctly different coordination. While the $\mathrm{Sc} 1$ atoms have only platinum neighbours in the form of a capped cube $(\mathrm{CsCl}$ slab within this intergrowth structure), the $\mathrm{Sc} 2$ atoms have a sandwich of two $\mathrm{Pt}_{3} \mathrm{Si}_{2}$ pentagons and further platinum and scandium neighbours capping the rectangular sites of the distorted pentagonal prism (Figure 7). Also here one observes distinct differences in the NMR spectroscopic data.

The carbides $\mathrm{Sc}_{3} T \mathrm{C}_{4}(T=\mathrm{Mn}, \mathrm{Fe}, \mathrm{Co}, \mathrm{Ni}, \mathrm{Ru}, \mathrm{Rh}, \mathrm{Os}$, Ir) [52, 62-66] have complex structural behavior. They crystallize with a pronounced subcell structure which was first refined for the cobalt compound [67]. Superstructure formation has then been observed from high-resolution single crystal X-ray data for $T=\mathrm{Co}, \mathrm{Rh}, \mathrm{Ir}, \mathrm{Ru}$, Os. Depending on the electron count, two different superstructure variants occur, which leads to a splitting of the two subcell scandium sites to three, respectively four sites in the two superstructures. Here we only discuss the subcell coordinations of $\mathrm{Sc}_{3} \mathrm{CoC}_{4}$ (Figure 7). The $\mathrm{Sc1}$ atoms have a sandwich-like coordination by two slightly elongated but planar $\mathrm{Co}_{2} \mathrm{C}_{4}$ hexagons with covalent $\mathrm{Sc}-\mathrm{C}$ bonding. Between the hexagons the scandium coordination sphere is completed by four scandium neighbours of the other type. The situation is different for $\mathrm{Sc} 2$. Here, two $\mathrm{CoC}_{2}$ fragments and one $\mathrm{C}_{2}$ pair coordinate in addition to six scandium neighbours, leading to a significantly different electronic situation for Sc2 as compared to Sc1. The two superstructure variants result from small, pair-wise shifts of the transition metal atoms off the subcell mirror planes. For further structural details on these carbides we refer to the literature.

\section{Applications of ${ }^{45} \mathrm{Sc}$ solid state NMR to structural issues in Sc-based intermetallic compounds}

\section{Local site symmetry, site multiplicity, and assignments in $\operatorname{ScAu} X(X=\mathrm{Si}, \mathrm{Ge}, \mathrm{Sn})$}

Figure 8 summarizes the ${ }^{45} \mathrm{Sc}$ MAS-NMR spectra of $\mathrm{ScAu} X$ compounds $(X=\mathrm{Si}, \mathrm{Ge}$, $\mathrm{Sn})$. The above-described differences in the Sc local site symmetries manifest themselves clearly in the breadth of the MAS spinning sidebands attributed to the non-central 
Im> <-> Im-1> Zeeman transitions. In cubic ScAuSn, the local electric field gradient vanishes at the scandium site, and no spinning sideband pattern is observed. In contrast, the local electric field gradient observed at the scandium site in ScAuGe gives rise to a spinning sideband pattern extending over a range of about $250 \mathrm{kHz}$, reflecting a fairly small $\mathrm{C}_{\mathrm{Q}}$ value on the order of $1 \mathrm{MHz}$. Finally, the above-described local distortion observed in ScAuSi gives rise to two crystallographically inequivalent Sc sites, characterized by two distinct resonance shifts (720 and 1169 ppm) and nuclear electric quadrupolar coupling constants. Evidently, the resonance at 720 ppm has the wider spinning sideband pattern, indicating a stronger quadrupolar interaction for this site than for the one at 1169 ppm. WIEN2k calculations indicate $\mathrm{C}_{\mathrm{Q}}=5.6$ and $3.8 \mathrm{MHz}$, for $\mathrm{Sc}(1)$ and $\mathrm{Sc}(2)$, respectively, suggesting the assignment of the 720 ppm peak to $\operatorname{Sc}(1)$ and of the 1169 ppm peak to $\mathrm{Sc}(2)$. In the present compound, this assignment can be tested independently by more sophisticated NMR experiments. While the Sc1 sites have six nearest silicon neighbours at a distance of $280 \mathrm{pm}$ and six nearest gold neighbours at a distance of $316 \mathrm{pm}$, the situation for the Sc2 sites is exactly the reverse one. As a result, Sc(1) experiences significantly stronger ${ }^{29} \mathrm{Si}^{45}{ }^{45} \mathrm{Sc}$ magnetic dipole-dipole coupling than $\mathrm{Sc}(2)$ as the strength of this interaction scales with the inverse cube of the internuclear distances. These dipolar couplings can be measured selectively using the technique of ${ }^{45} \mathrm{Sc}\left\{{ }^{29} \mathrm{Si}\right\}$ rotational echo double resonance (REDOR) studies on isotopically enriched material. This experiment measures the attenuation of the ${ }^{45} \mathrm{Sc}$ NMR signal that is caused by ${ }^{29} \mathrm{Si}$ spin inversion during the magic angle spinning rotor cycle. As Figure 9 indicates the Sc site giving rise to the signal at $720 \mathrm{ppm}$ shows the stronger attenuation in this experiment, indicating the stronger stronger ${ }^{29} \mathrm{Si}^{4}{ }^{45} \mathrm{Sc}$ magnetic dipole-dipole coupling, thereby confirming the above assignment to the $\operatorname{Sc}(1)$ site.

\section{Superstructure formation and site characterization in ScAgSn}

Figures 10 and 11 illustrates the use of ${ }^{45} \mathrm{Sc}$ advanced high-resolution solid state NMR methodology for differentiating subtly different Sc environments having strong nuclear electric quadrupolar coupling interactions. The example chosen here concerns the detection and characterization of the superstructure formed in ScAgSn [49]. As described above this superstructure is derived from ScPtSn by a structural distortion leading to three distinct Sc sites with subtly different local environments. While the structural in- 
equivalence of these scandium sites is indeed evident in the ${ }^{45} \mathrm{Sc}$ MAS-NMR spectrum (top trace of Figure 10), the resolution is poor owing to the small differences in magnetic shielding and the presence of substantial second-order quadrupolar broadening effects affecting the lineshapes of the central $|1 / 2><->|-1 / 2>$ Zeeman transitions. As shown in Figure 10, the anisotropic quadrupolar broadening can be successfully removed by the 2-D TQMAS technique, leading to three well-resolved resonances in the isotropic (F1) dimension. Furthermore, as these signals are well-separated in the 2D contour plot, the individual anisotropically broadened "subspectra" can be observed for each site, and reliable spectral parameters extracted via lineshape simulations (Figures 11a-c). Based on these individual component fits, a simulation the overall MAS-NMR lineshape is possible (Figure 11d). Finally, the three signals were unambiguously assigned by comparing their experimental $\mathrm{C}_{\mathrm{Q}}$ and $\eta_{\mathrm{Q}}$ parameters with theoretically calculated values.

\section{Defect characterization and quantification in non-stoichiometric systems}

${ }^{45} \mathrm{Sc}$ MAS-NMR spectra have proven to be sensitive to the effects of incomplete site occupancies in a variety of $\operatorname{Sc} T_{\mathrm{x}} X_{\mathrm{y}}$ compounds. For example, the ${ }^{45} \mathrm{Sc}$ resonances in $\mathrm{ScNi}_{1.54} \mathrm{Sn}$ are broadened significantly by second-order quadrupolar interactions, owing to the local electric field gradients produced by the Ni vacancies in the coordination environments [51]. In the compound with nominal composition $\mathrm{ScNiSi}_{3}$ two distinct $\mathrm{Sc}$ signals at 588 and 618 ppm are observed, having signal area fractions of $70 \%$ and $30 \%$, respectively (see Figure 12). This result is easily understood in view of the deficient Ni occupancy of $92 \%$. As the first coordination sphere of the Sc atoms includes $4 \mathrm{Ni}$ atoms, the vacancy population of $8 \%$ implies that the statistical probability of Sc to have one neighboring Ni vacancy is $32 \%$. This prediction is in excellent agreement with the NMR result, if the 618 ppm peak is assigned to Sc near a Ni vacancy [54]. Similar results have been obtained for the half-Heusler phases $\operatorname{ScTSb}(T=\mathrm{Ni}, \mathrm{Pd}, \mathrm{Pt})$. The Ni- and $\mathrm{Pd}$ compounds are slightly metal deficient, corresponding to vacancy concentrations of about $14 \%$ for the Ni- and $3.5 \%$ for the Pd-site. The ${ }^{45}$ Sc MAS-NMR spectra of these compounds are shown in Figure 13; in addition a comparison is made between rapidly quenched and annealed samples. For the annealed $\mathrm{ScNi}_{0.86} \mathrm{Sb}$ sample two resonances are observed at 1025 ppm (56 \%) and 1014 ppm (46\%), which can be assigned to the regu- 
lar Sc site and the Sc site near a Ni vacancy, respectively. In the annealed $\mathrm{ScPd}_{0.965} \mathrm{Sb}$ sample the analogous signals are observed at 1070 ppm (79 \%) and 1065 ppm (21\%). Since the $\mathrm{Sc}$ atoms are coordinated by $4 \mathrm{Ni}(\mathrm{Pd})$ atoms in the structure a statistical distribution of vacancies implies that about $56 \%$ and $14 \%$ of the scandium species would have a neighboring vacancy in the $\mathrm{Ni}$ compound and the $\mathrm{Pd}$ compound, respectively. Given the experimental uncertainties of the compositions, the populations measured by NMR are in reasonable agreement with these predictions. The quenched $\mathrm{ScTSb}(T=\mathrm{Ni}$, Pd) samples are showing a third resonance at 1003 ppm, and $1056 \mathrm{ppm}$ respectively. The disappearance of these signals upon annealing clearly suggests that they can be assigned to scandium atoms having a disordered $T$-environment. This type of disorder can be created if some $T$ atoms are shifted into the free tetrahedral voids in the structure, thereby breaking the local cubic symmetry and creating a third spectral feature arising from the ${ }^{45} \mathrm{Sc}$ nuclei in the vicinity of such defects. For ScPtSb all sites were found to be fully occupied by XRD, which is also reflected by a single sharp resonance in the ${ }^{45} \mathrm{Sc}$ MAS NMR spectrum.

\section{Polymorphism and phase transitions in $\mathrm{Sc}_{3} T \mathrm{CC}_{4}(T=\mathrm{Ni}, \mathrm{Co}, \mathrm{Ru}, \mathrm{Rh}, \mathrm{Os}, \mathrm{Ir})$}

Figure 14 shows the ${ }^{45} \mathrm{Sc}$ MAS NMR spectra of a series of the scandium metal carbides with composition $\mathrm{Sc}_{3} \mathrm{TC}_{4}(T=\mathrm{Ni}, \mathrm{Co}, \mathrm{Ru}, \mathrm{Rh}$, Os, and $\mathrm{Ir}$ ) [52]. The two distinct scandium sites observed for $\mathrm{Sc}_{3} \mathrm{CoC}_{4}$, described above, are easily identified according to their population ratio $\operatorname{Sc}(1) / \mathrm{Sc}(2)=1: 2$. The large $(1000 \mathrm{ppm})$ difference in their isotropic magnetic shielding parameters indicates a huge difference in Knight shift, suggesting that the $\operatorname{Sc}(2)$ site experiences a much higher unpaired conduction electron density near the Fermi edge than the Sc(1) site. Inspection of Figure 14 indicates this feature to be general for all of the compounds of the present series. The room temperature spectra of $\mathrm{Sc}_{3} \mathrm{RhC}_{4}$ and $\mathrm{Sc}_{3} \mathrm{IrC}_{4}$ show three resonances, consistent with the splitting of the $\mathrm{Sc}(2)$ site into two distinct environments. For $\mathrm{Sc}_{3} \mathrm{RuC}_{4}$ and $\mathrm{Sc}_{3} \mathrm{OsC}_{4}$ phase transitions below room temperature give rise to new superstructures having four crystallographically distinct $\mathrm{Sc}$ sites. As shown in Figure 15 for $\mathrm{Sc}_{3} \mathrm{OsC}_{4}$, this phase transition can be monitored well by temperature dependent NMR spectra. The simulation of the low temperature experiment is shown in Figure 16. In this low-temperature phase, the unique $\mathrm{Sc}(2)$ position of the high temperature phase split into two signals at $754 \mathrm{ppm}$ and 639 
ppm. Likewise the unique Sc(1) position of the high-temperature phase splits into two signals at 444 and 405 ppm. An additional resonance observed near 706 ppm, which is reminiscent of the $\operatorname{Sc}(2)$ site observed in the high-temperature phase, signifies some residual disorder. These results illustrate the potential and power of variable-temperature ${ }^{45} \mathrm{Sc}$ MAS-NMR to monitor phase transitions and superstructure formation in this class of compounds.

\section{Summary and Conclusions}

In summary, the results of the present review suggest that ${ }^{45} \mathrm{Sc}$ NMR is a powerful probe for structural investigations of Sc-based intermetallic compounds. First of all, the excellent ability of NMR to differentiate between crystallographically inequivalent sites in a quantitative fashion makes this method very useful for confirming proposed crystal structure solutions from x-ray diffractometry. Assignment problems (which site to be attributed to which resonance in the case of equal populations) can be addressed by comparing the experimental quadrupolar coupling parameters with quantum theoretically computed values or by advanced dipolar re-coupling experiments exploiting differences in the magnitude of heteronuclear magnetic dipole-dipole interactions with other nuclear isotopes in the first or second coordination sphere. Furthermore, the quality and validity of a proposed crystal structure can be tested by comparing the nuclear electric quadrupolar coupling constants and asymmetry parameters computed directly from the crystal structure data with those measured from NMR spectroscopy. If larger deviations between experimental and calculated values are observed, the proposed structure solution either may not be valid or there is a discrepancy between the single crystal used on the diffractometer and the bulk polycrystalline material used in the NMR analysis. Such structure validation by NMR becomes especially important for compounds based on elements with very similar scattering power, for materials presenting single crystals of low quality or twinning, and - of course - for those cases where only polycrystalline or amorphous samples are available. Finally, the ability of NMR to resolve locally distinct environments allow in-depth characterizations of order/disorder phenomena including (1) occupancy deficiencies in non-stoichiometric compounds, (2) site multiplicities produced by positional disordering, and (3) phase transitions/superstructure formation. 
A final issue concerns the interpretation of the internal NMR interaction parameters in terms of geometrical and electronic structure information. Owing to the multiple structural and electronic origins in intermetallic compounds we have found it is generally impossible to correlate nuclear electric quadrupolar coupling constants and/or asymmetry parameters with simple geometric distortion parameters, even within closely related series of isotypic compounds. Given the complex bonding situation in ternary Scbased intermetallic compounds this finding is not unexpected and thus emphasizes the necessity of resorting to quantum mechanical calculations for being able to understand the sizes and symmetries of the electric field gradients in these compounds.

Similar conclusions hold for the isotropic magnetic shielding constant, $\delta_{\mathrm{ms}}$ iso . For all the compounds we have measured thus far, the large positive resonance displacements measured against an ionic reference standard $\left(\mathrm{Sc}^{3+}\right.$ in aqueous solution) suggests that the Knight shift contributions are dominant. This is not unexpected if the Sc atoms make significant contributions to the density of states in the vicinity of the Fermi-level. At the present time, reliable ab-initio calculations of ${ }^{45} \mathrm{Sc}$ Knight shifts are not available for ternary Sc-based intermetallic compounds. Thus, for developing the understanding of the magnetic shielding effects observed in the present compounds any further, in-depth theoretical analyses of the electronic structures of these materials as well as their variations as a function of composition in closely related materials are required.

This work was financially supported by the Deutsche Forschungsgemeinschaft through SPP 1166 Lanthanoidspezifische Funktionalitäten in Molekül und Material and by the NRW Graduate School of Chemistry. We thank our past PhD students Thomas Harmening, Sebastian Peter, Christian Voigt, and Constanze Fehse, on whose dissertations this review is based. Thanks are also due to Daniel Mohr, Adel Al Alam, Samir Matar, Long Zhang, Rolf-Dieter Hoffmann, and Leo van Wüllen, for contributing to this work and for useful discussions.

\section{References}

[1] K. A. Gschneidner, Jr. in Scandium, Its Occurrence, Chemistry, Physics, Metallurgy, Biology and Technology (C. T. Horowitz, ed.), Academic Press, London 1978, pp. 152-332. 
[2] B. Ya Kotur, E. Gratz, Scandium Alloy Systems and Intermetallics, in Handbook of the Physics and Chmistry of Rare Earths (K. A. Gschneidner, Jr, L. Eyring, eds.), Elsevier Amsterdam 1999, ch. 175.

[3] K. Sato, S. Takeda, S. Fukuda, T. Minamisono, M. Tanigaki, T. Miyake, Y. Maruyama, K. Matsuta, M. Fukuda, Y. Nojiri, Z. Naturforsch. 1998, 53a, 549.

[4] A. A. Vashman, Y. B. Muravlev, E. G. Il'in, Y. A. Buslaev, Dokl. Akad. Nauk. 1999, 365, 777.

[5] R. Blinc, A. Gregorovič, B. Zalar, R. Pirc, S. G. Lushnikov, Phys. Rev. B. 2000, $61,253$.

[6] D. Riou, F. Fayon, D. Massiot, Chem. Mater. 2002, 14, 2416.

[7] C. Tien, E. V. Charnaya, S. Y. Sun, R. R. Wu, S. N. Ivanov, E. N. Khazanov, Phys. Stat. Sol. 2002, 233b, 222.

[8] C. T. Brigden, D. Thompsett, C. D. Williams, Dalton Trans. 2004, 2829.

[9] V. V. Laguta, M. D. Glinchuk, I. P. Bykov, R. Blinc, B. Zalar, Phys. Rev. B. 2004, 69, 054103.

[10] S. R. Miller, A. M. Z. Slawin, P. Wormald, P. A. Wright, J. Solid State Chem. 2005, 178, 1738.

[11] D. Khabibulin, K. Romanenko, M. Zuev, O. Lapina, Magn. Reson. Chem. 2007, $45,962$.

[12] C. S. Lue, C. N. Kuo, T. H. Su, G. J. Redhammer, Phys. Rev. B 2007, 75, 014426.

[13] N. Kim, J. F. Stebbins, S. Quartieri, R. Oberti, Am. Mineral. 2007, 92, 1875.

[14] D. Mohr, A. S. S. de Camargo, C. C. de Araujo, H. Eckert, J. Mater. Chem. 2007, $17,3733$.

[15] C. T. Brigden, C. D. Williams, D. Apperley, Inorg. Mater. 2007, 43, 758.

[16] H. Huang, C.-H. Hsieh, N. Kim, J. Stebbins, F. Prinz, Solid State Ionics 2008, 179, 1442.

[17] D. Mohr, A. S. S. de Camargo, J. F. Schneider, T. B. de Queiroz, H. Eckert, E. R. Botero, D. Garcia, J. A. Eiras, Solid State Sci. 2008, 10, 1401.

[18] S. P. Shafi, M. W. Kotyk, L. M. D. Cranswick, V. K. Michaelis, S. Kroeker, M. Bieringer, Inorg. Chem. 2009, 48, 10553.

[19] K. E. Kelsey, J. F. Stebbins, D. M. Singer, G. E. Brown Jr., J. L. Mosenfelder, P. D. Asimow, Geochim. Cosmochim. Acta 2009, 73, 3914.

[20] P. Jain, H. J. Avila-Paredes, C. Gapuz, S. Sen, S. Kim, J. Phys. Chem. C 2009, $113,6553$.

[21] N. Kim, J. F. Stebbins, Chem. Mater. 2009, 21, 309.

[22] S. Balamurugan, U. Ch. Rodewald, T. Harmening, L. van Wüllen, D. Mohr, H. Eckert, R. Pöttgen, Z. Naturforsch. 2010, 65b, 13.

[23] H. J. Avila-Paredes, P. Jain, S. Sen, S. Kim, Chem. Mater. 2010, 22, 893. 
[24] A. C. A. Jayasundera, A. A. Finch, P. Wormald, P. Lightfoot, Chem. Mater. 2008, 20,6810 .

[25] A. Y. H. Lo, V. Sudarsan, S. Sivakumar, F. van Veggel, R. W. Schurko, J. Am. Chem. Soc. 2007, 129, 4687.

[26] S. Giri, H. Nakamura, T. Kohara, Phys. Rev. B. 2005, 72, 132404.

[27] N. Büttgen, A. Zymara, C. Kegler, V. Tsurkan, A. Loidl, Phys. Rev. B 2006, 73, 132409.

[28] Y. Miyake, S. Suzuki, Y. Kojima, K. Kikuchi, K. Kobayashi, S. Nagase, M. Kainosho, Y. Achiba, Y. Maniwa, K. Fisher, J. Phys. Chem. 1996, 100, 9579.

[29] M. Krause, F. Ziegs, A. A. Popov, L. Dunsch, Chem. Phys. Chem. 2007, 8, 537.

[30] H. Okimoto, W. Hemme, Y. Ito, T. Sugai, R. Kitaura, H. Eckert, H. Shinohara, NANO 2008, 3, 21.

[31] X. Wang, T. Zuo, M. M. Olmstead, J. C. Duchamp, T. E. Glass, F. Cromer, A. L. Bach, H. C. Dorn, J. Am. Chem. Soc. 2006, 128, 8884.

[32] S. Yang, A. A. Popov, C. Chen, L. Dunsch, J. Phys. Chem. C 2009, 113, 7616.

[33] S. E. Ashbrook, S. Wimperis, J. Magn. Reson. 2000, 147, 238.

[34] K. J. Pike, R. P. Malde, S. E. Ashbrook, J. McManus, S. Wimperis, Solid State Nucl. Magn. Reson. 2000, 16, 203.

[35] S. E. Ashbrook, S. Wimperis, J. Magn. Res. 2002, 156, 269.

[36] T. Bräuniger, K. Ramaswamy, P. K. Madhu, Chem. Phys. Lett. 2004, 383, 403.

[37] H. Park, I. Bull, L. Peng, V. G. Young, C. P. Grey, J. B. Parise, Chem. Mater. 2004, 16, 5350.

[38] N. Kim, C.-H. Hsieh, J. F. Stebbins, Chem. Mater. 2006, 18, 3855.

[39] A. J. Rossini, R. W. Schurko, J. Am. Chem. Soc. 2006, 128, 10391.

[40] P.-J. Chu, B. C. Gerstein, H. D. Yang, R. N, Shelton, Phys. Rev B 1988, 37, 1796.

[41] T. Koyama, H. Sugita, S. Wada, K. Tsutsumi, J. Phys. Soc. Jpn. 1999, 68, 2326.

[42] C. S. Lue, R. F. Liu, Y. F. Fu, C. Cheng, H. D. Yang, Phys. Rev. B 2008, 77, 115130.

[43] M. Oku, T. Shishido, T. Shinohara, T. Fukuda, Q. Sun, Y. Kawazoe, K. Wagatsuma, J. Alloys Compd. 2002, 339, 317.

[44] M. Oku, T. Shishido, T. Shinohara, Q. Sun, Y. Kawazoe, K. Nakajima, K. Wagatsuma, J. Solid State Chem. 2004, 177, 457.

[45] J. J. Balbach, M. S. Conradi, R. G. Barnes, D. S. Sibirtsev, A. V. Skripov, Phys. Rev. B 1999, 60, 966.

[46] P. C. M. M. Magusin, W. P. Kalisvaart, P. H. L. Notten, R. A. van Santen, Chem. Phys. Lett. 2008, 456, 55. 
[47] C. P. Sebastian, H. Eckert, S. Rayaprol, R.-D. Hoffmann, R. Pöttgen, Solid State Sci. 2006, 8, 560.

[48] C. P. Sebastian, C. Fehse, H. Eckert, R.-D. Hoffmann, R. Pöttgen, Solid State Sci. 2006, 8, 1386.

[49] C. P. Sebastian, L. Zhang, C. Fehse, R.-D. Hoffmann, H. Eckert, R. Pöttgen, Inorg. Chem. 2007, 46, 771.

[50] C. P. Sebastian, L. Zhang, H. Eckert, R. Pöttgen, Z. Naturforsch. 2007, 62b, 173.

[51] C. P. Sebastian, H. Eckert, R. Pöttgen, Solid State Sci. 2007, 9, 357.

[52] L. Zhang, C. Fehse, H. Eckert, C. Vogt, R.-D. Hoffmann, R. Pöttgen, Solid State Sci. 2007, 9, 699.

[53] T. Harmening, C. P. Sebastian, L. Zhang, C. Fehse, H. Eckert, R. Pöttgen, Solid State Sci. 2008, 10, 1395.

[54] Th. Harmening, H. Eckert, D. Johrendt, R. Pöttgen, Solid State Sci. 2008, 10, 544.

[55] T. Harmening, H. Eckert, R. Pöttgen, Solid State Sci. 2009, 11, 900.

[56] T. Harmening, A. Al Alam, S. F. Matar, H. Eckert, R. Pöttgen, Solid State Sci. 2009, 11, 1239.

[57] T. Harmening, L. van Wüllen, H. Eckert, U. Ch. Rodewald, R. Pöttgen, Z. Anorg. Allg. Chem. 2010, 636, in press.

[58] T. Harmening, D. Mohr, H. Eckert, A. Al Alam, S. F. Matar, R. Pöttgen, Z. Anorg. Allg. Chem. 2010, 636, in press.

[59] A. Medek, J. S. Harwood, L. Frydman, J. Am. Chem. Soc. 1995, 117, 12779.

[60] P. Blaha, K. Schwarz, K. H. Madsen, D. Kvasnicka, J. Luiz, WIEN2k, An Augmented Plane Wave and Local Orbitals Program for Calculating Crystal Properties, K. H. Schwarz, Techn. Univ. Wien, Austria 2001, ISBN 3-9501031-2.

[61] D. Massiot, F. Fayon, M. Capron, I. King, S. Le Calve, B. Alonso, J. O Durand, B. Bujoli, Z. Gan, G. Hoatson, Magn. Reson. Chem. 2002, 40, 70.

[62] R.-D. Hoffmann, R. Pöttgen, W. Jeitschko, J. Solid State Chem. 1992, 99, 134.

[63] Chr. Vogt, R.-D. Hoffmann, R. Pöttgen, Solid State Sci. 2005, 7, 1003.

[64] B. Rohrmoser, G. Eickerling, M. Presnitz, W. Scherer, V. Eyert, R.-D. Hoffmann, U. Ch. Rodewald, C. Vogt, R. Pöttgen, J. Am. Chem. Soc. 2007, 129, 9356.

[65] C. Vogt, R.-D. Hoffmann, U. Ch. Rodewald, G. Eickerling, M. Presnitz, V. Eyert, W. Scherer, R. Pöttgen, Inorg. Chem. 2009, 48, 6436.

[66] W. Scherer, C. Hauf, M. Presnitz, E.-W. Scheidt, G. Eickerling, V. Eyert, R.-D. Hoffmann, U. Ch. Rodewald, A. Hammerschmidt, C. Vogt, R. Pöttgen, Angew. Chem. 2010, 122, 1623; Angew. Chem. Int. Ed. 2010, 49, 1578.

[67] A. O. Tsokol', O. I. Bodak, E. P. Marusin, Sov. Phys. Crystallogr. 1986, 31, 466. 


\section{Figure Captions}
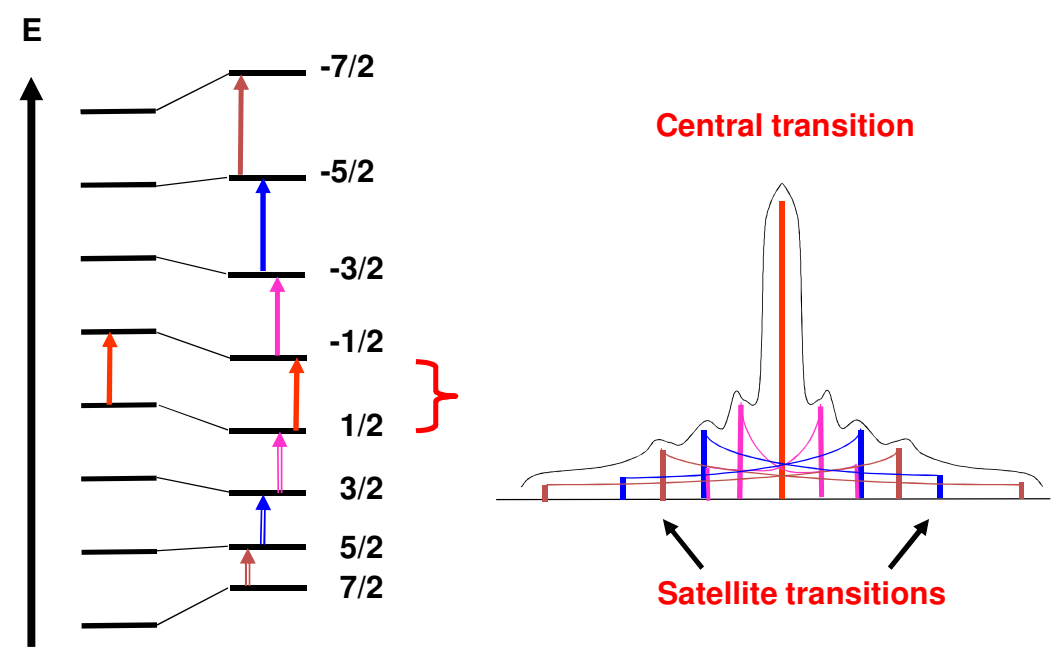

Fig. 1 Zeeman energy level diagram for a spin-7/2 nucleus affected by first-order quadrupolar perturbations and resulting lineshape under static conditions. 


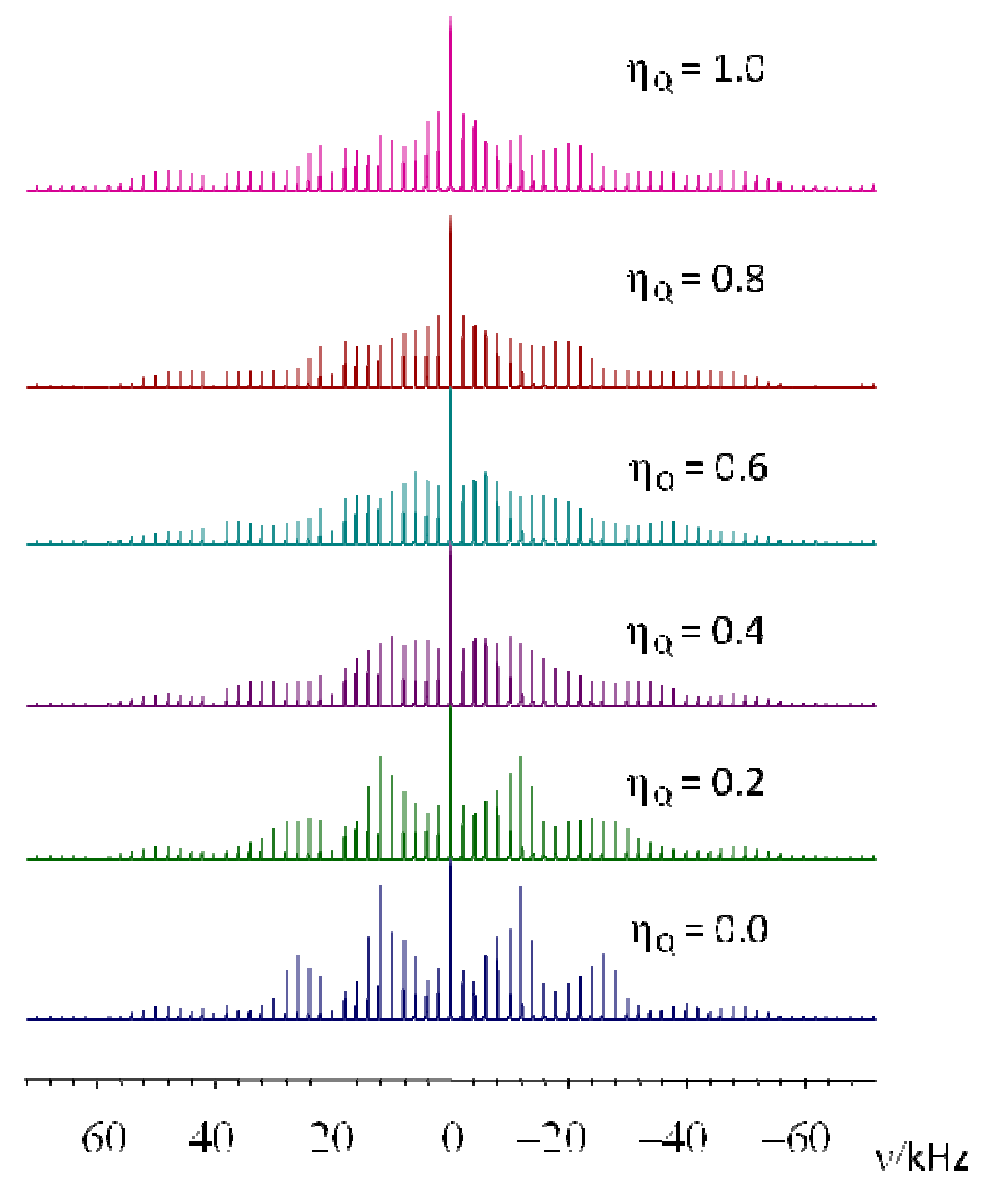

Fig. 2 Spinning sideband intensity distribution calculated via the SIMPSON programme for a ${ }^{45} \mathrm{Sc}$ nucleus having a nuclear electric quadrupolar coupling constant of $4.0 \mathrm{MHz}$ for different values of the asymmetry parameter $\eta_{\mathrm{Q}}$. 

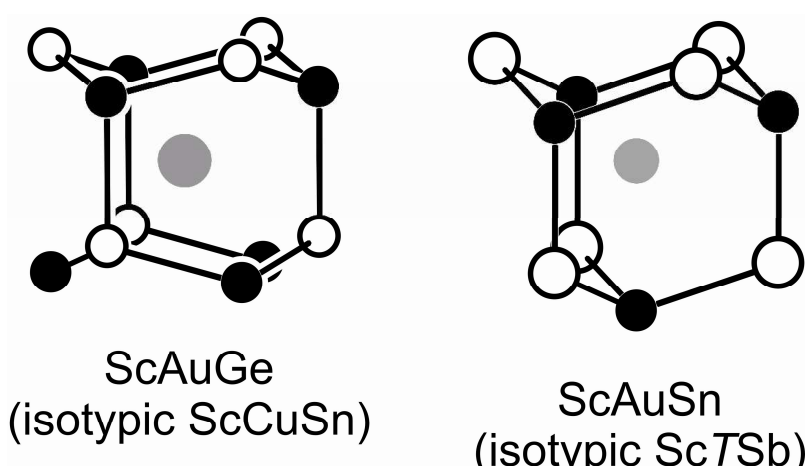

ScAuSn (isotypic ScTSb)
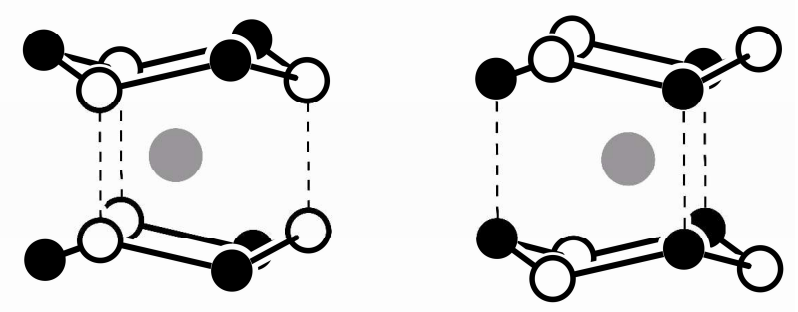

Sc1 in ScAuSi

Fig. 3 Coordination of the scandium atoms in the structures of ScAuGe, ScAuSn, and ScAuSi. Scandium, transition metal, and silicon (germanium, tin) atoms are drawn as medium grey, black filled, and open circles, respectively. Isotypic compounds are indicated. The coordinating $T_{3} X_{3}$ hexagons are emphasized. For details see text. 


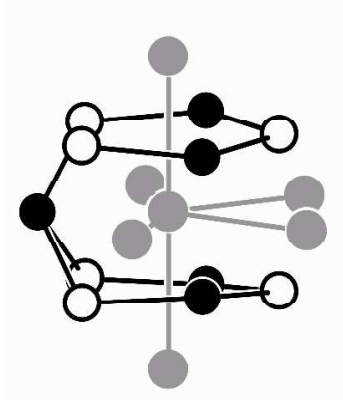

ScPdSn

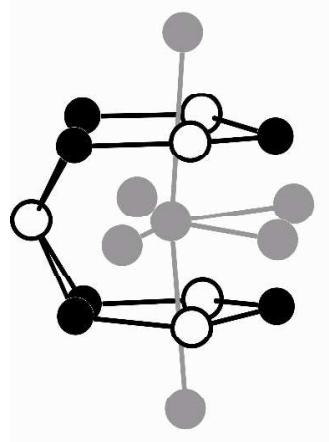

Sc2 in ScAgSn

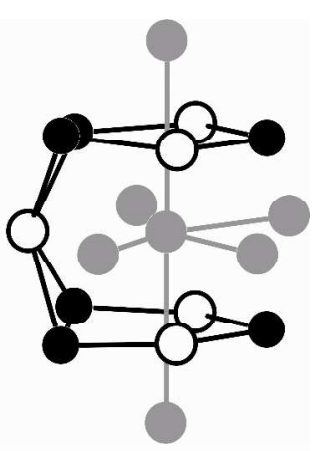

Sc1 in ScAgSn

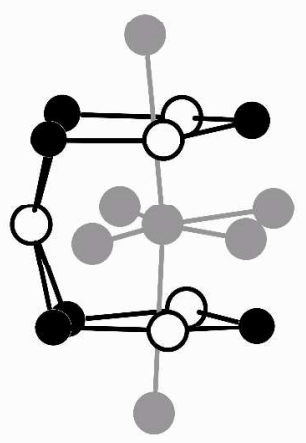

Sc3 in ScAgSn

Fig. 4 Coordination of the scandium atoms in the structures of ScPdSn and ScAgSn. Scandium, transition metal, and tin atoms are drawn as medium grey, black filled, and open circles, respectively. For details see text. 


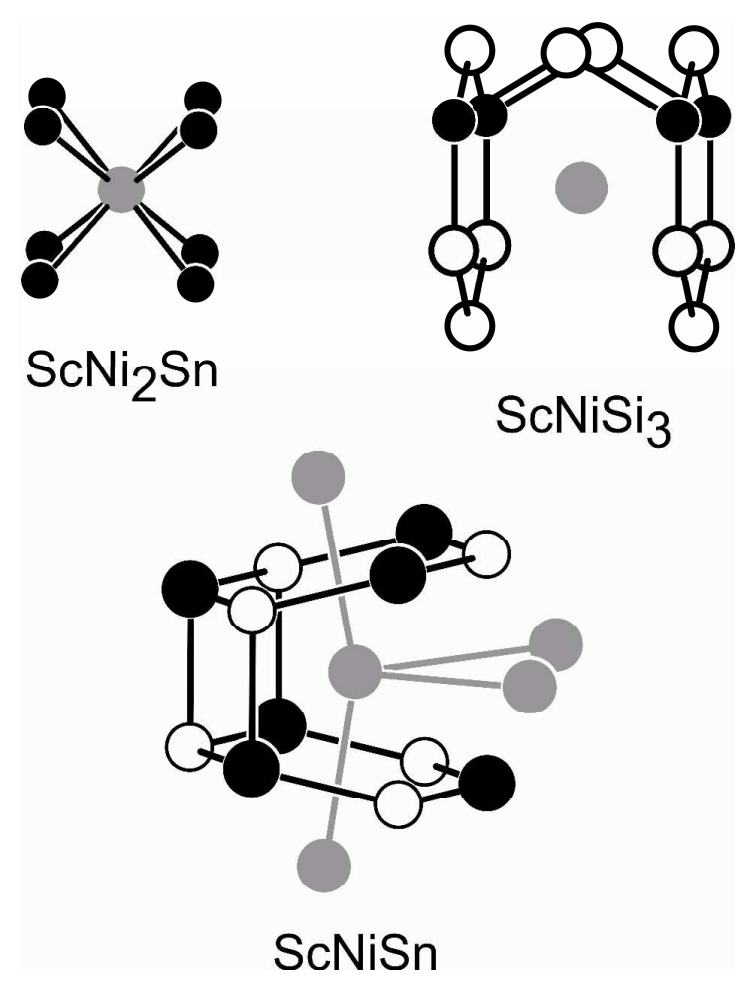

Fig. 5 Coordination of the scandium atoms in the structures of $\mathrm{ScNi}_{2} \mathrm{Sn}, \mathrm{ScNiSi}_{3}$, and ScNiSn. Scandium, transition metal, and tin (silicon) atoms are drawn as medium grey, black filled, and open circles, respectively. For details see text. 

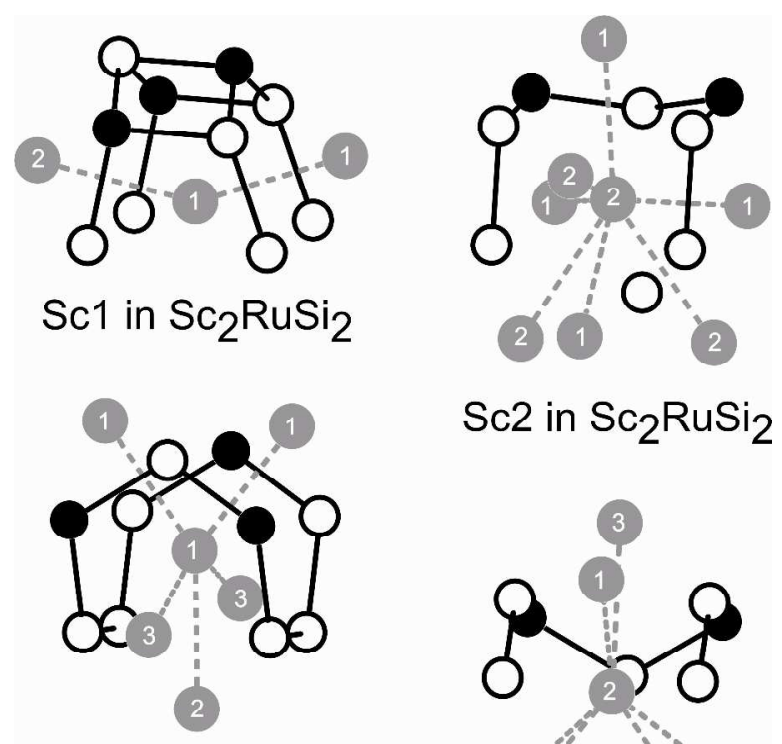

$\mathrm{Sc} 2$ in $\mathrm{Sc}_{2} \mathrm{RuSi}_{2}$
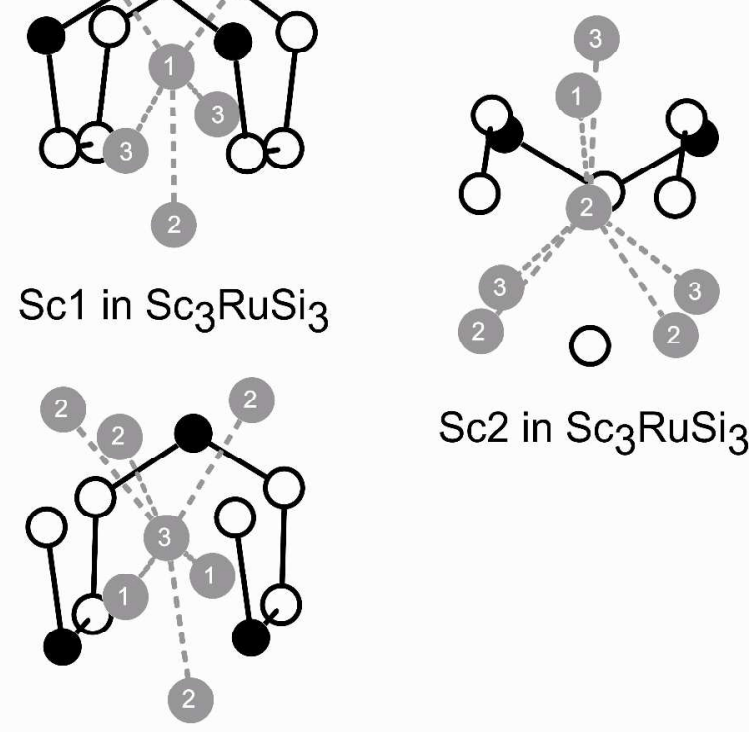

$\mathrm{Sc} 2$ in $\mathrm{Sc}_{3} \mathrm{RuSi}_{3}$

$\mathrm{Sc} 3$ in $\mathrm{Sc}_{3} \mathrm{RuSi}_{3}$

Fig. 6 Coordination of the scandium atoms in the silicides $\mathrm{Sc}_{2} \mathrm{RuSi}_{2}$ and $\mathrm{Sc}_{3} \mathrm{RuSi}_{3}$. Scandium, ruthenium, and silicon atoms are drawn as medium grey, black filled, and open circles, respectively. The crystallographically different scandium sites are indicated. For details see text. 


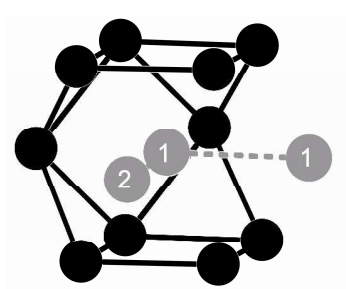

$\mathrm{Sc} 1$ in $\mathrm{Sc}_{4} \mathrm{Pt}_{7} \mathrm{Si}_{2}$
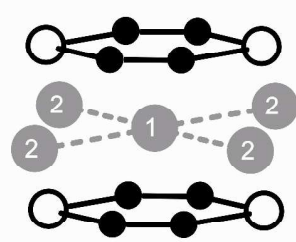

$\mathrm{Sc} 1$ in $\mathrm{Sc}_{3} \mathrm{CoC}_{4}$

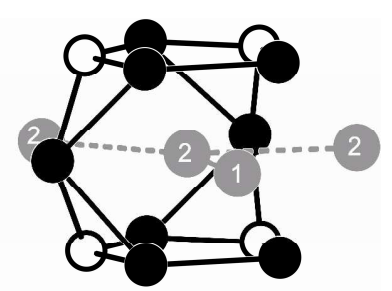

$\mathrm{Sc} 2$ in $\mathrm{Sc}_{4} \mathrm{Pt}_{7} \mathrm{Si}_{2}$

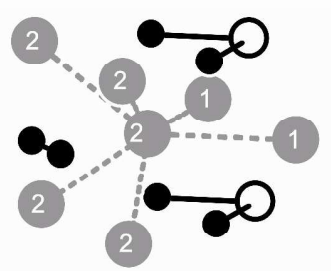

$\mathrm{Sc} 2$ in $\mathrm{Sc}_{3} \mathrm{CoC}_{4}$

Fig. 7 Coordination of the scandium atoms in $\mathrm{Sc}_{4} \mathrm{Pt}_{7} \mathrm{Si}_{2}$ and the subcell of $\mathrm{Sc}_{3} \mathrm{CoC}_{4}$. Scandium, platinum, and silicon atoms for $\mathrm{Sc}_{4} \mathrm{Pt}_{7} \mathrm{Si}_{2}$ are drawn as medium grey, black filled, and open circles, respectively. The cobalt and carbon atoms in $\mathrm{Sc}_{3} \mathrm{CoC}_{4}$ are drawn as open and filled circles, respectively. The crystallographically different scandium sites are indicated. For details see text. 


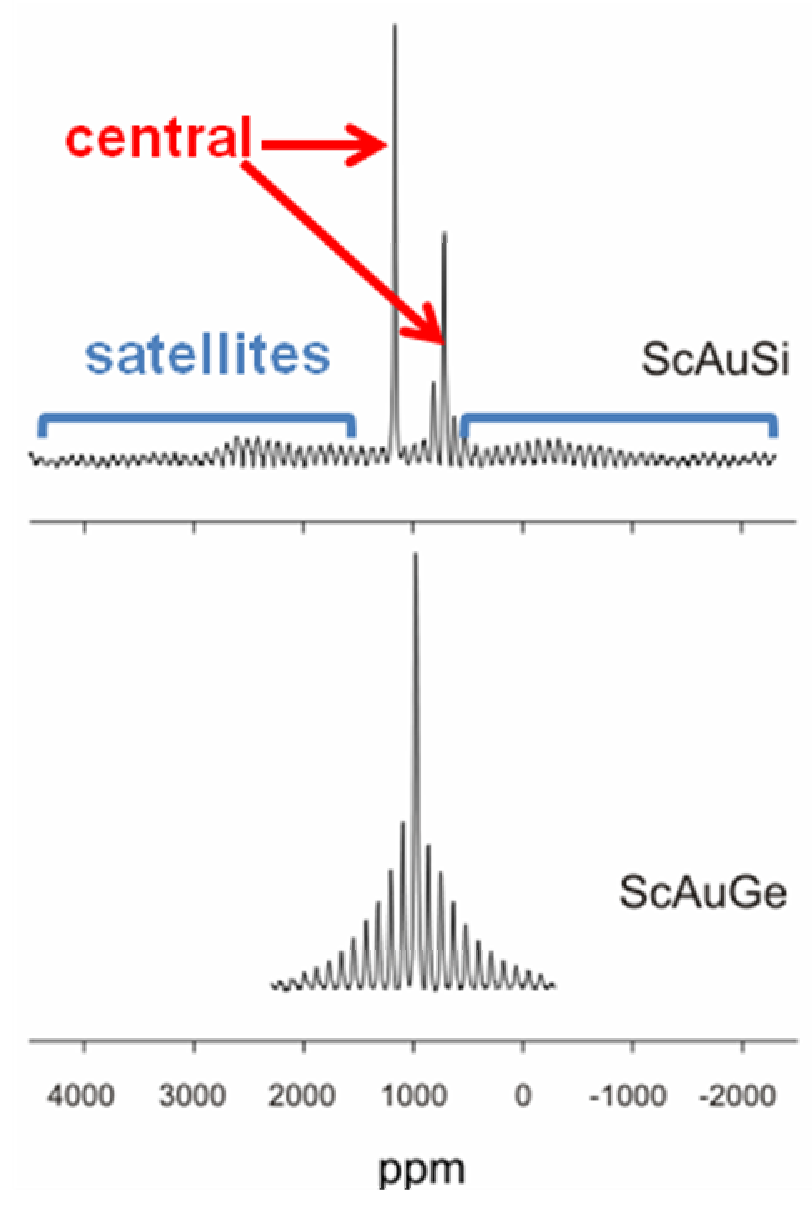

ScAuSn

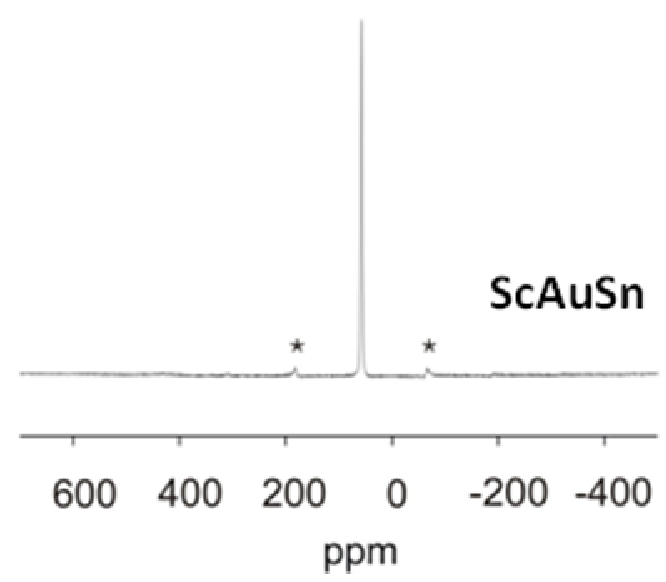

Fig. ${ }^{45}$ Sc MAS-NMR spectra of ScAuSi, ScAuGe, and ScAuSn. The central I1/2><-> $\mid-1 / 2>$ and the satellite transitions arising from the other $|\mathrm{m}\rangle<->|\mathrm{m}-1\rangle$ transitions are indicated in the figure (modified from [50] and [47] with permission of Zeitschrift für Naturforschung and Elsevier). 


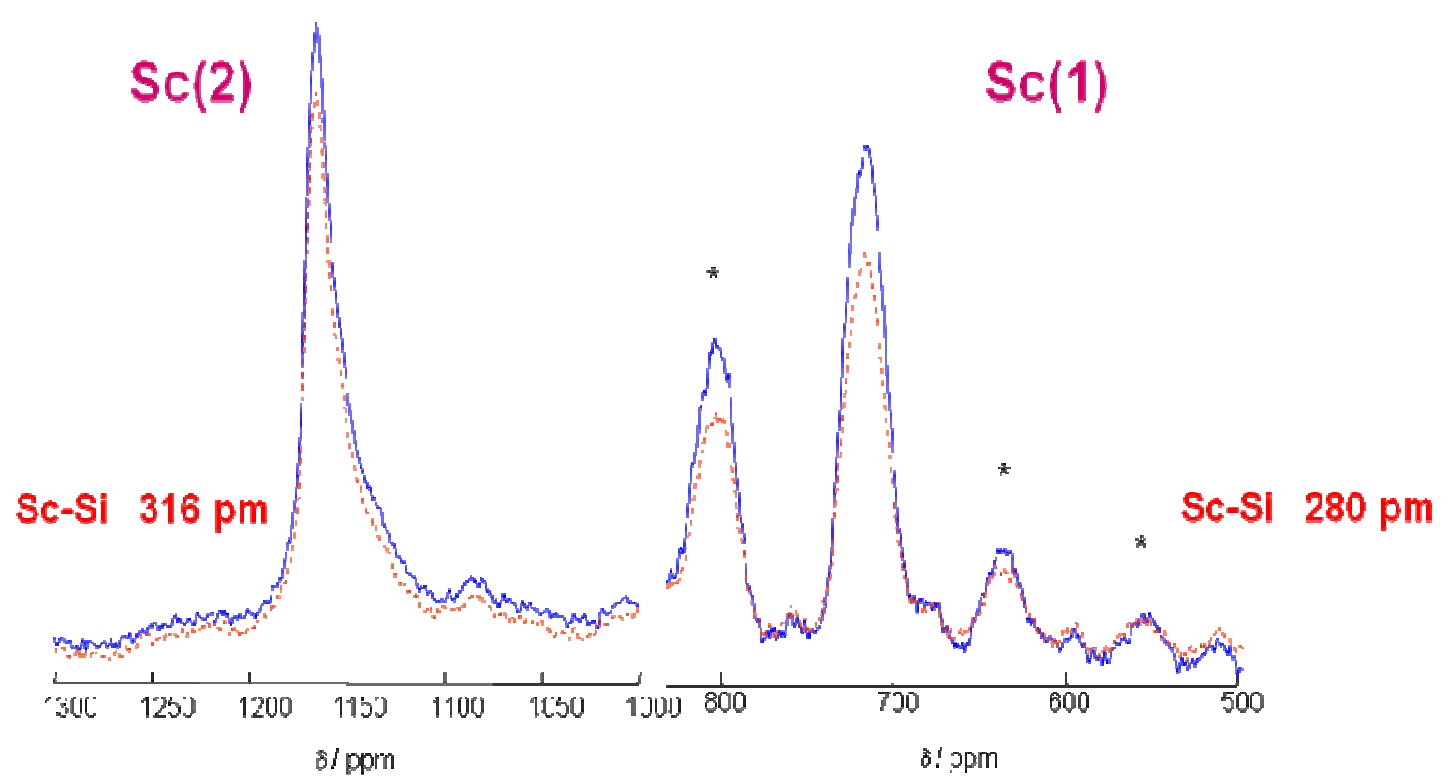

Fig. $9{ }^{45} \mathrm{Sc}\left\{{ }^{29} \mathrm{Si}\right\}$ rotational echo double resonance (REDOR) studies on isotopically enriched $\mathrm{ScAuSi}$. Blue and red curves reflect the regular and the dipolarely attenuated MAS-NMR signals of the $\mathrm{Sc}(1)$ and the $\mathrm{Sc}(2)$ sites respectively. The dipolar evolution time used in this experiment was $1.5 \mathrm{~ms}$ (modified reproduction from [50] with permission of Zeitschrift für Naturforschung). 


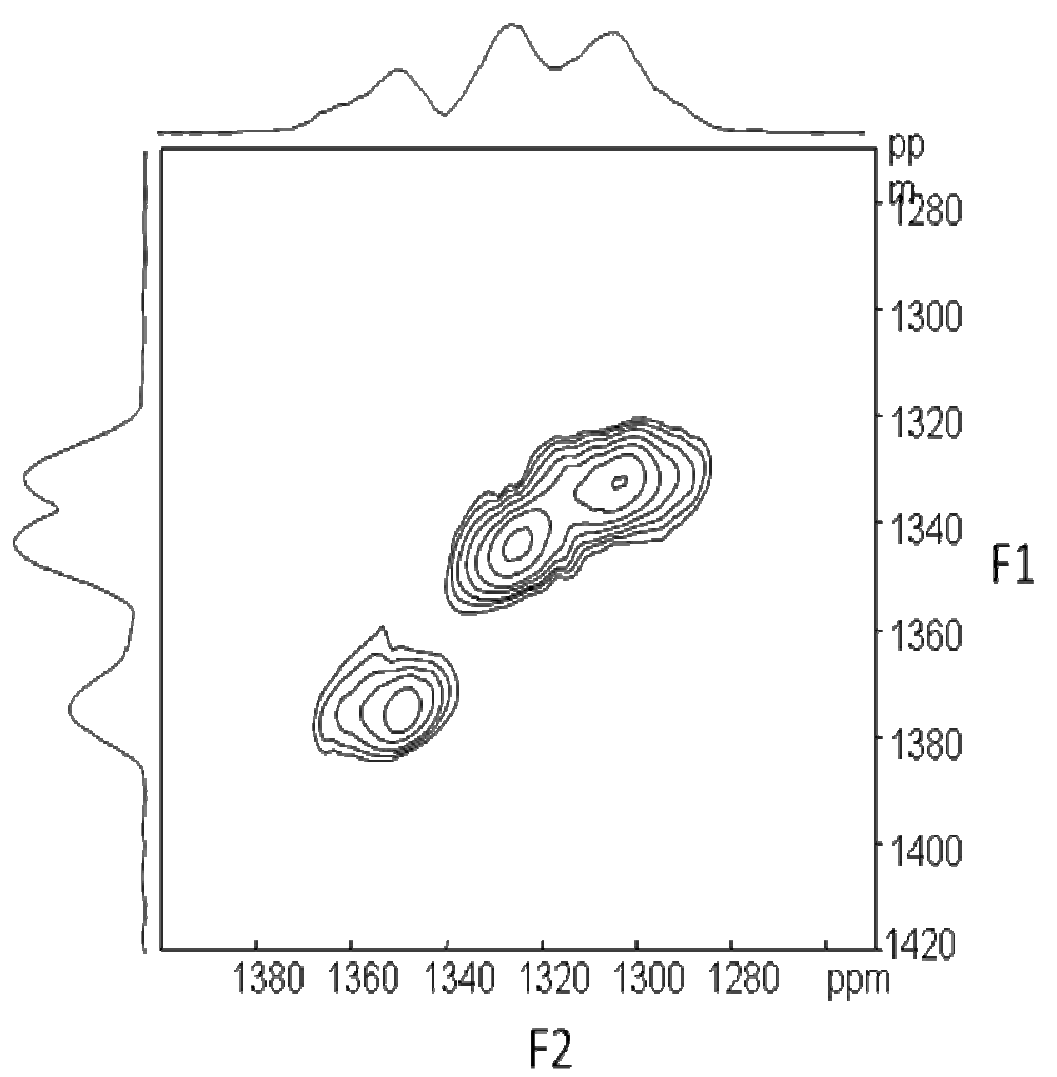

Fig. 10 Two-dimensional ${ }^{45} \mathrm{Sc}$ triple-quantum MAS NMR spectrum of ScAgSn. The horizontal dimension (F2) corresponds to the standard MAS-NMR spectrum, while in the vertical dimension (F1) the resolution is enhanced due to the removal of secondorder quadrupolar broadening (modified reproduction from [49] with permission of ACS). 

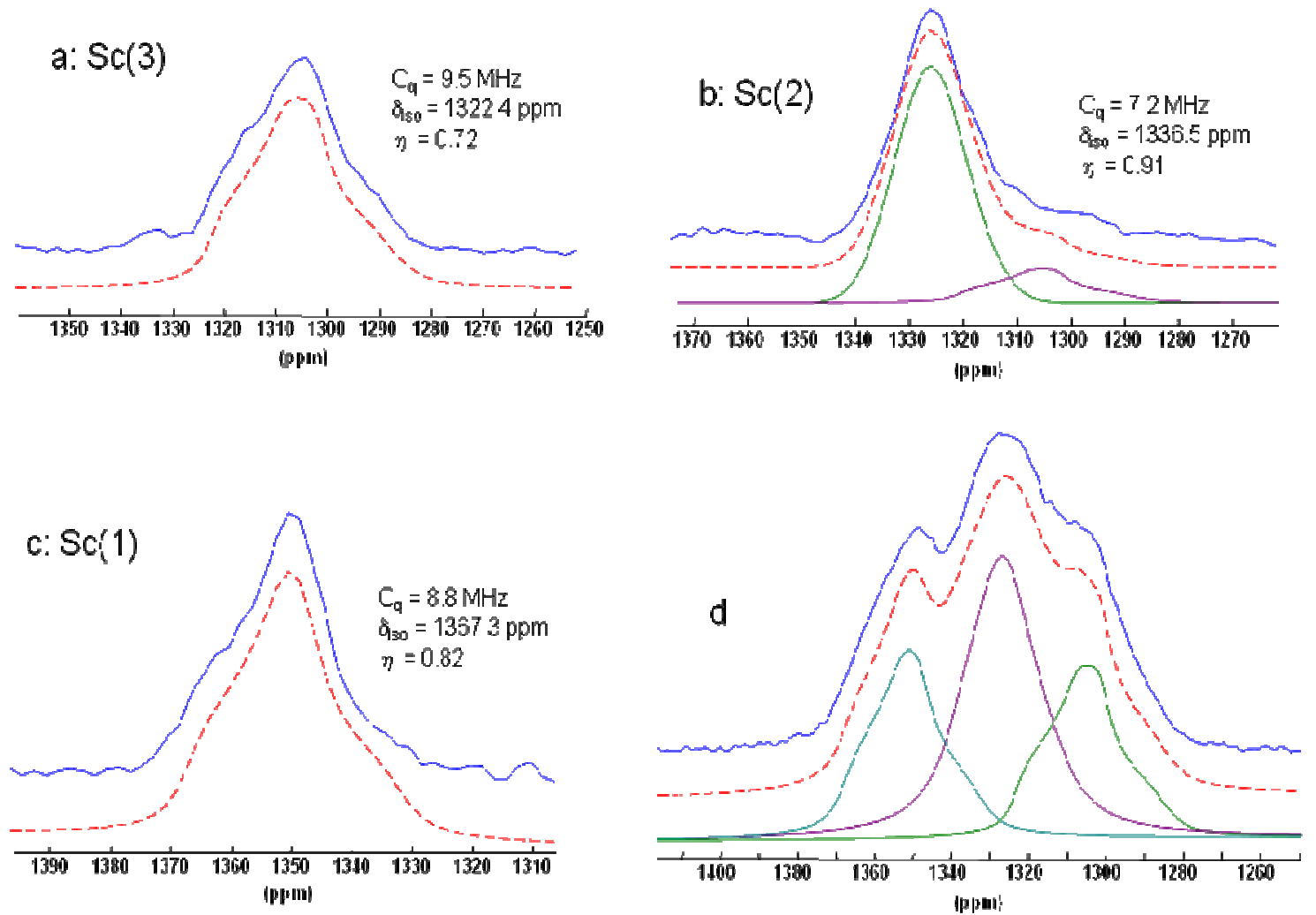

Fig. 11 One-dimensinonal ${ }^{45} \mathrm{Sc}$ MAS-NMR spectra of ScAgSn. (a-c): Individual subspectra observed for the three distinct Sc sites in the anisotropic dimension of the ${ }^{45} \mathrm{Sc}$ TQMAS experiment and their corresponding spectral simulations; (d) standard MASNMR spectrum and its simulation based on the individual lineshape parameters extracted from the plots a-c (modified reproduction from [49] with permission of ACS). 


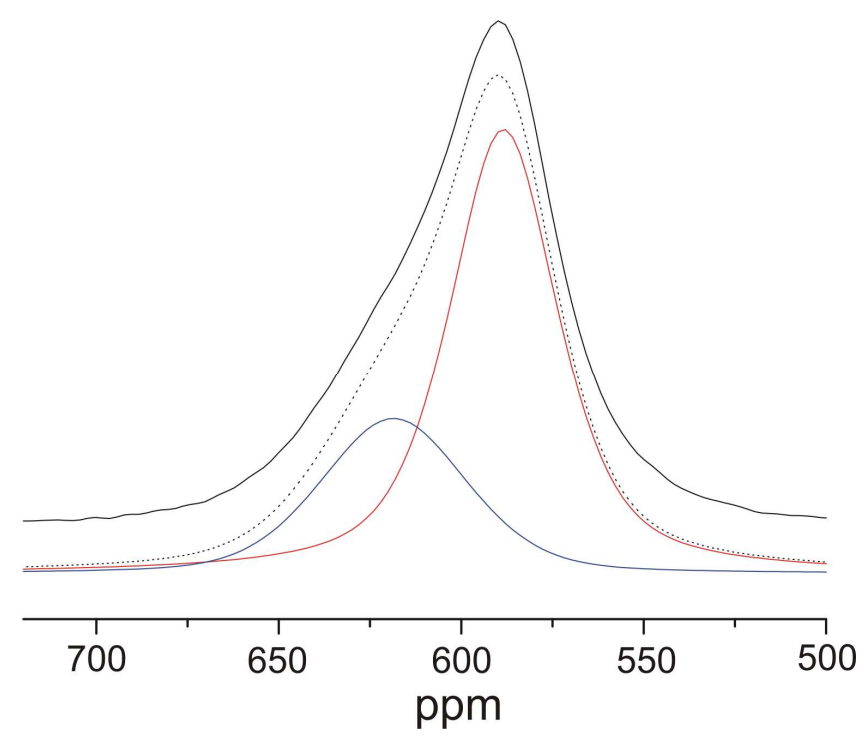

Fig. 12 Central resonance of the ${ }^{45} \mathrm{Sc}$ MAS-NMR spectrum of $\mathrm{ScNiSi}_{3}$ and deconvolution into two Gaussian components (reproduction from [54] with permission from Elsevier). 

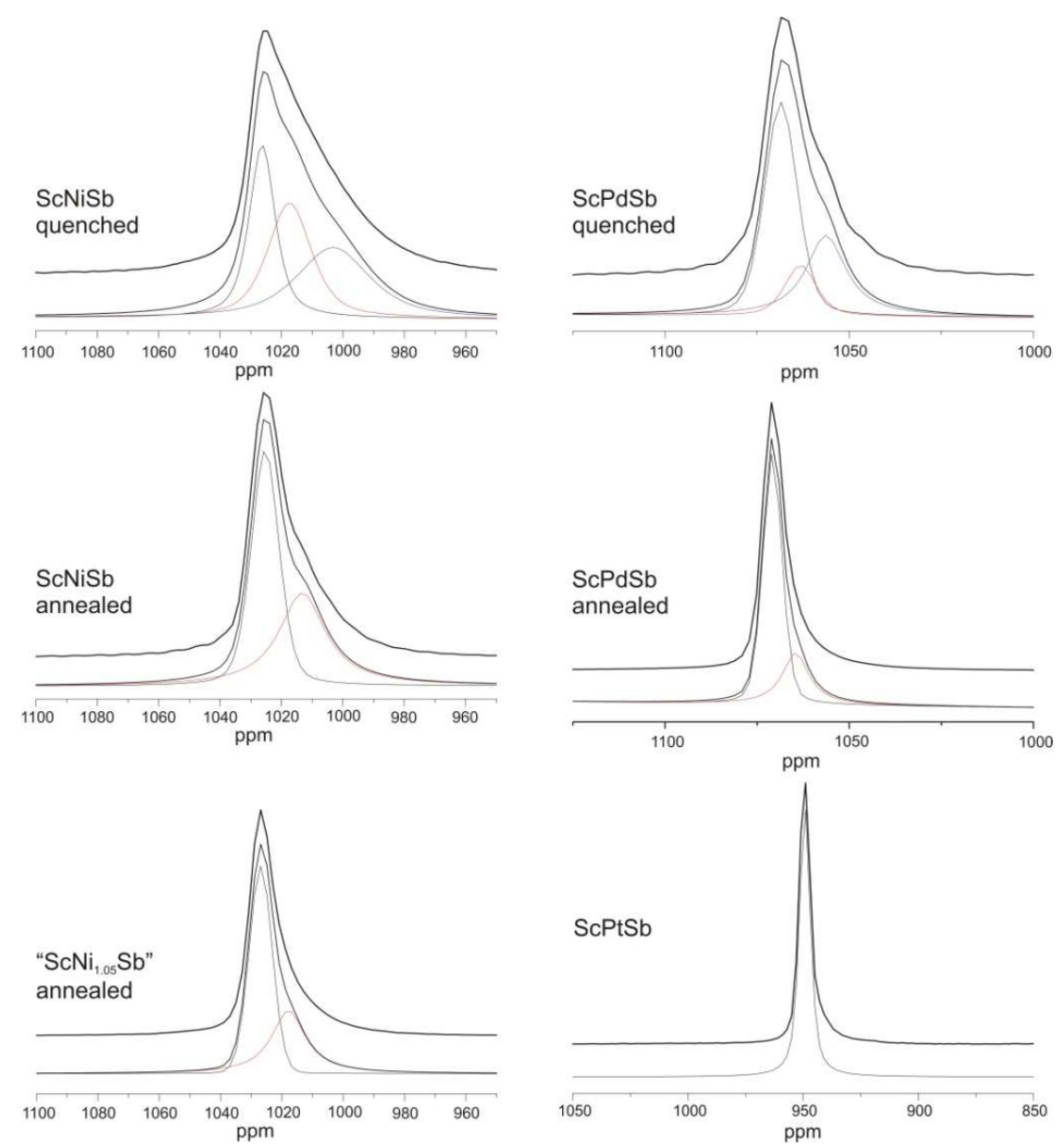

Fig. $13{ }^{45} \mathrm{Sc}$ MAS-NMR spectra of ScTSb compounds $(T=\mathrm{Ni}, \mathrm{Pd}, \mathrm{Pt})$ and their lineshape simulations (reproduction from [55] with permission from Elsevier). 


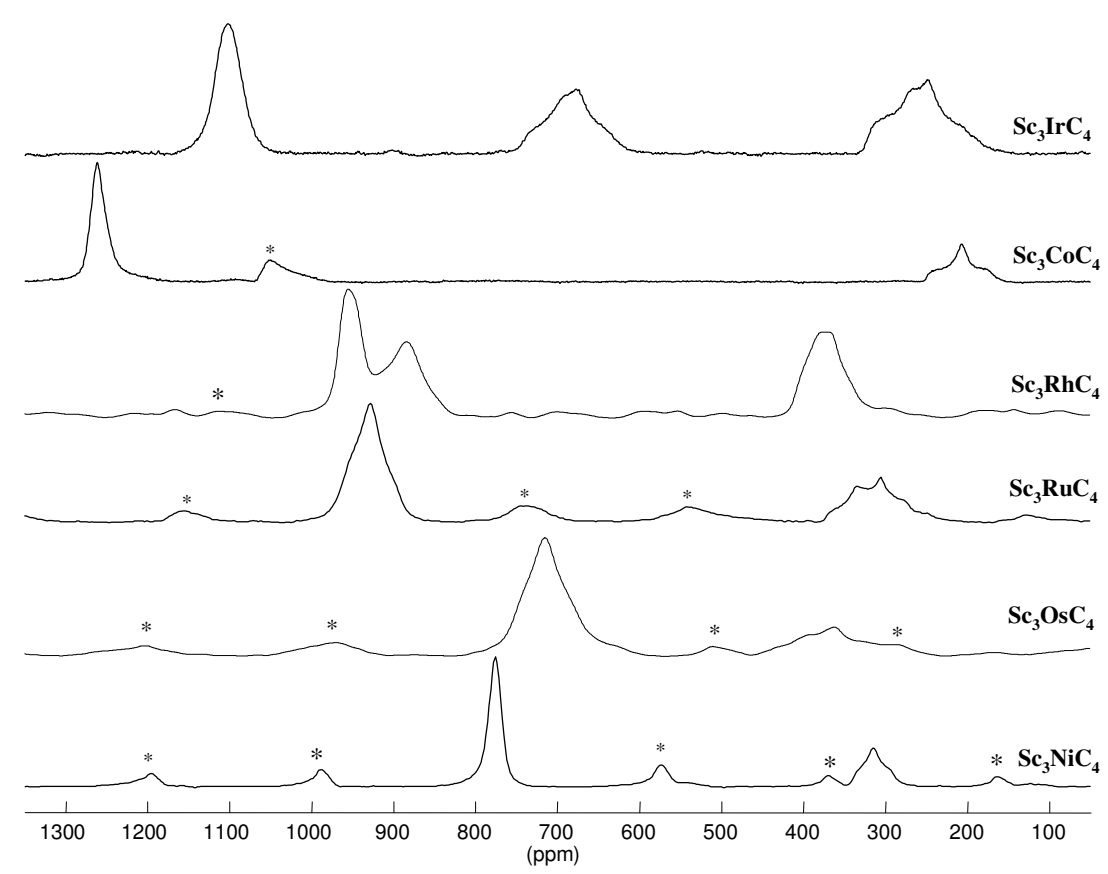

Fig. $14{ }^{45} \mathrm{Sc}$ MAS-NMR spectra of $\mathrm{Sc}_{3} T \mathrm{CC}_{4}$ compounds (reproduction from [52] with permission from Elsevier).

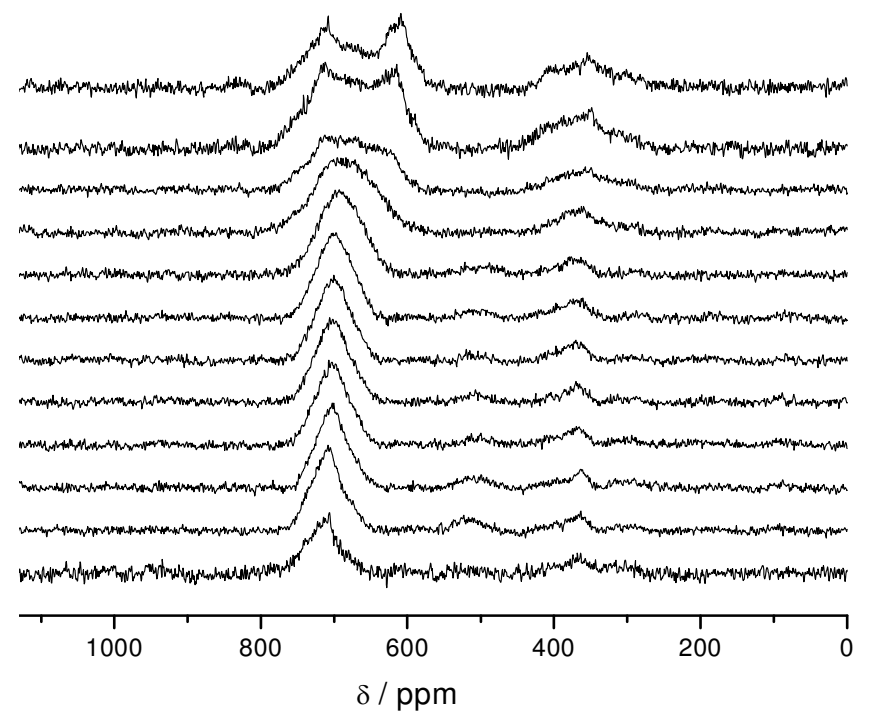

Fig. 15 Variable temperature ${ }^{45} \mathrm{Sc}$ MAS NMR of $\mathrm{Sc}_{3} \mathrm{OsC}_{4}$. From bottom to top the temperatures correspond to 286, 276, 266, 257, 256, 247, 242, 237, 227, 217, 207, and 197 $\mathrm{K}$. 


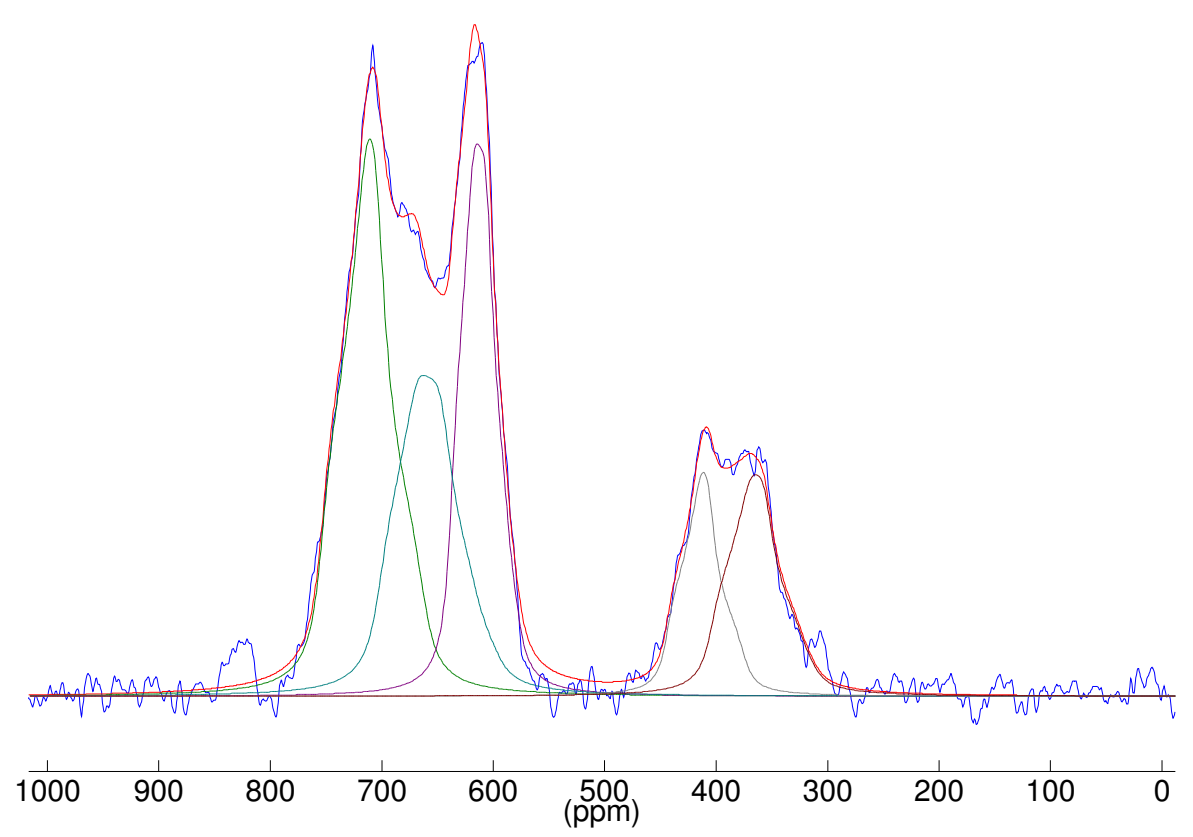

Fig. $16191 \mathrm{~K}{ }^{45} \mathrm{Sc}$ MAS-NMR spectrum of the LT phase of $\mathrm{Sc}_{3} \mathrm{OsC}_{4}$. Black curves show simulations of the spectral component belonging to five distinct $\mathrm{Sc}$ sites. These sites are simulated with the parameters $\delta_{\mathrm{ms}}{ }^{\text {iso }}, \mathrm{C}_{\mathrm{Q}}$ and $\eta$ : $754 \mathrm{ppm}, 14.1 \mathrm{MHz}, 0.82 ; 639$ ppm, 11.7 MHz, 0.59; 444 ppm, 12.2 MHz, 0.81; 405 ppm, 14.1 MHz, 0.70; 706 ppm, 15.8 $\mathrm{MHz}, 0.52$. 